\title{
Measurement of Cosmic Microwave Background Polarization Power Spectra from Two Years of BICEP Data
}

\section{Citation}

Chiang, H. Cynthia, Peter A. R. Ade, Denis Barkats, John O. Battle, Evan M. Bierman, James J. Bock, C. Darren Dowell, and et al. 2010. Measurement of cosmic microwave background polarization power spectra from two years of BICEP data. The Astrophysical Journal 711, no. 2: 1123-1140.

\section{Published Version}

doi:10.1088/0004-637X/711/2/1123

\section{Permanent link}

http://nrs.harvard.edu/urn-3:HUL.InstRepos:11129150

\section{Terms of Use}

This article was downloaded from Harvard University's DASH repository, and is made available under the terms and conditions applicable to Other Posted Material, as set forth at http:// nrs.harvard.edu/urn-3:HUL.InstRepos:dash.current.terms-of-use\#LAA

\section{Share Your Story}

The Harvard community has made this article openly available.

Please share how this access benefits you. Submit a story.

Accessibility 


\title{
MEASUREMENT OF COSMIC MICROWAVE BACKGROUND POLARIZATION POWER SPECTRA FROM TWO YEARS OF BICEP DATA
}

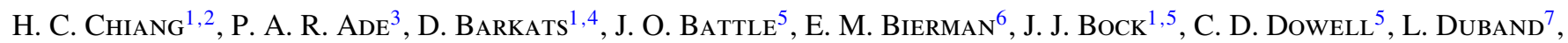

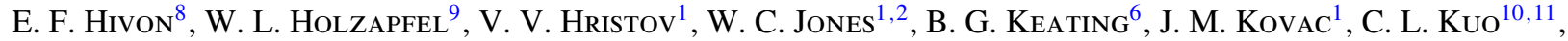 \\ A. E. Lange ${ }^{1,5,15}$, E. M. Leitch ${ }^{12}$, P. V. Mason ${ }^{1}$, T. Matsumura ${ }^{1}$, H. T. Nguyen ${ }^{5}$, N. Ponthieu $^{13}$, C. Pryke $^{12}$, S. Richter ${ }^{1}$,

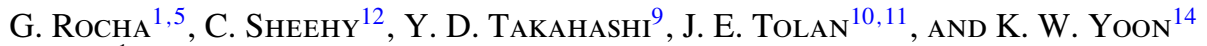 \\ ${ }^{1}$ Department of Physics, California Institute of Technology, Pasadena, CA 91125, USA \\ ${ }^{2}$ Department of Physics, Princeton University, Princeton, NJ 08544, USA \\ ${ }^{3}$ Department of Physics and Astronomy, University of Wales, Cardiff, CF24 3YB, Wales, UK \\ ${ }^{4}$ National Radio Astronomy Observatory, Santiago, Chile \\ 5 Jet Propulsion Laboratory, Pasadena, CA 91109, USA \\ ${ }^{6}$ Department of Physics, University of California at San Diego, La Jolla, CA 92093, USA \\ ${ }^{7}$ SBT, Commissariat à l'Energie Atomique, Grenoble, France \\ ${ }^{8}$ Institut d'Astrophysique de Paris, Paris, France \\ ${ }^{9}$ Department of Physics, University of California at Berkeley, Berkeley, CA 94720, USA \\ ${ }^{10}$ Stanford University, Palo Alto, CA 94305, USA \\ ${ }^{11}$ Kavli Institute for Particle Astrophysics and Cosmology (KIPAC), Sand Hill Road 2575, Menlo Park, CA 94025, USA \\ ${ }^{12}$ University of Chicago, Chicago, IL 60637, USA \\ ${ }^{13}$ Institut d'Astrophysique Spatiale, Université Paris-Sud, Orsay, France \\ ${ }^{14}$ National Institute of Standards and Technology, Boulder, CO 80305, USA \\ Received 2009 June 15; accepted 2010 January 29; published 2010 February 22
}

\begin{abstract}
Background Imaging of Cosmic Extragalactic Polarization (BICEP) is a bolometric polarimeter designed to measure the inflationary $B$-mode polarization of the cosmic microwave background (CMB) at degree angular scales. During three seasons of observing at the South Pole (2006 through 2008), BiceP mapped 2\% of the sky chosen to be uniquely clean of polarized foreground emission. Here, we present initial results derived from a subset of the data acquired during the first two years. We present maps of temperature, Stokes $Q$ and $U, E$ and $B$ modes, and associated angular power spectra. We demonstrate that the polarization data are self-consistent by performing a series of jackknife tests. We study potential systematic errors in detail and show that they are sub-dominant to the statistical errors. We measure the $E$-mode angular power spectrum with high precision at $21 \leqslant \ell \leqslant 335$, detecting for the first time the peak expected at $\ell \sim 140$. The measured $E$-mode spectrum is consistent with expectations from a $\Lambda \mathrm{CDM}$ model, and the $B$-mode spectrum is consistent with zero. The tensor-to-scalar ratio derived from the $B$-mode spectrum is $r=0.02_{-0.26}^{+0.31}$, or $r<0.72$ at $95 \%$ confidence, the first meaningful constraint on the inflationary gravitational wave background to come directly from CMB $B$-mode polarization.
\end{abstract}

Key words: cosmic background radiation - cosmology: observations - gravitational waves - inflation polarization

Online-only material: color figure

\section{INTRODUCTION}

One of the cornerstones in our current understanding of cosmology is the theory of inflation. Inflation addresses several major shortcomings of the standard big bang model, resolving the flatness and horizon problems and explaining the origin of structure; however, the theory has yet to be unambiguously confirmed by observational evidence.

Numerous experiments have demonstrated that the cosmic microwave background (CMB) is an extremely effective tool for studying the early universe. Precision measurements of the temperature anisotropies now span a wide range of angular scales (Jones et al. 2006; Reichardt et al. 2009; Nolta et al. 2009; Friedman et al. 2009; Sievers et al. 2009; Brown et al. 2009) and have yielded tight constraints on a model of the universe in which the energy content is dominated by a cosmological constant and cold dark matter $(\Lambda \mathrm{CDM})$.

The polarization anisotropies of the CMB provide even more insight into the history of the universe, potentially encoding in-

\footnotetext{
${ }^{15}$ Sadly, Andrew Lange passed away shortly before the publication of this article.
}

formation from long before the moment of matter-radiation decoupling. The primary source of $\mathrm{CMB}$ polarization is Thomson scattering of the local quadrupole of the photonbaryon fluid sourced by density fluctuations. The resulting partial polarization has no handedness and is called the gradient or " $E$-mode" by analogy to curl-free electric fields. The $\Lambda \mathrm{CDM}$ parameters that predict the temperature spectrum also predict the shape of the $E$-mode spectrum with almost no additional information (reionization enhances power at the largest angular scales). $E$-mode polarization was first detected by DASI (Kovac et al. 2002) and has since been measured by many other experiments (Leitch et al. 2005; Montroy et al. 2006; Sievers et al. 2007; Wu et al. 2007; Bischoff et al. 2008). The acoustic peaks in the polarization spectra have been measured to high precision in both the TE spectrum (Piacentini et al. 2006; Nolta et al. 2009 ) and, more recently, directly in the $E E$ spectrum (Pryke et al. 2009; Brown et al. 2009), providing further support for our basic understanding of CMB physics.

Inflation predicts the existence of a stochastic gravitational wave background, created during the initial accelerated expansion of the universe, which imparts a unique imprint on the $\mathrm{CMB}$ 
at the surface of last scattering (Polnarev 1985). In addition to producing $E$-mode polarization, gravitational waves also induce a curl or " $B$-mode" in the polarization anisotropies (Seljak \& Zaldarriaga 1997; Kamionkowski et al. 1997). The inflationary $B$-mode signal is expected to peak at degree angular scales (multipole moment $\ell \sim 100$ ) with an amplitude determined by the energy scale of inflation. Because density fluctuations at the surface of last scattering create only $E$-mode polarization, a detection of the $B$-mode signal would be strong evidence that inflation occurred (see, e.g., Dodelson et al. 2009).

The inflationary $B$-mode amplitude is parameterized by the tensor-to-scalar ratio $r$, and the most restrictive published upper limit, $r<0.22$ (95\% confidence), comes from measurements of large-scale temperature anisotropies in combination with baryon acoustic oscillation and Type Ia supernova data (Komatsu et al. 2009). However, the constraints from temperature anisotropies are ultimately limited by cosmic variance, and lowering the $r$ limit further requires direct polarization measurements. Currently, limits from polarization are still far worse than those from temperature-for example, assuming $\Lambda \mathrm{CDM}$ parameters that are fixed at Wilkinson Microwave Anisotropy Probe (WMAP) best-fit values, the WMAP $B$-mode spectrum constrains $r<6$ at $95 \%$ confidence. The results reported in this paper provide upper limits on the $B$-mode signal that are an order of magnitude more stringent than those set by WMAP.

Background Imaging of Cosmic Extragalactic Polarization (BICEP) is a microwave polarimeter that has been designed specifically to probe the $B$-mode of CMB polarization at degree angular scales. The instrument observed from the South Pole between 2006 January and 2008 December. A detailed description of the BICEP instrument characterization procedures is given in a companion paper, Takahashi et al. (2010). In this paper, we report initial CMB polarization results from the 2006 and 2007 observing seasons.

\section{THE BICEP INSTRUMENT}

A complete description of the BICEP instrument is available in Yoon et al. (2006), and only a brief summary is given here. The BICEP receiver consists of a two-lens refracting telescope coupled to a focal plane of 49 orthogonal pairs of polarizationsensitive bolometers (PSBs; Jones et al. 2003). The PSB pairs are divided between 25 that observe at $100 \mathrm{GHz}$ and 24 at $150 \mathrm{GHz}$ (two of the $150 \mathrm{GHz}$ PSB pairs were reconfigured for $220 \mathrm{GHz}$ operation in late 2006 and were subsequently not used for CMB analysis). The angular resolution at 100 and $150 \mathrm{GHz}$ is 0.93 and 0.60 , respectively, and the instantaneous field of view is $18^{\circ}$. The entire focal plane and optics assembly is housed in an upward-looking cryostat with toroidal liquid nitrogen and liquid helium tanks. The clean optical path and azimuthal symmetry minimize instrumental polarization systematics.

The receiver is supported in an azimuth-elevation mount with a third degree of rotational freedom about the boresight. The mount is located on the top floor of the Dark Sector Laboratory (89.99 S, 44.65 W) at the Amundsen-Scott South Pole station, a site with excellent atmospheric transparency and stability at millimeter wavelengths as well as outstanding infrastructure. The telescope penetrates through the roof and is sealed to the building with a flexible environmental enclosure, leaving most of the instrument accessible in a warm lab setting.

The $24 \mathrm{hr}$ visibility of the target field from the South Pole enables uninterrupted observation and deep integration. BICEP's primary CMB field lies within the "Southern Hole,"

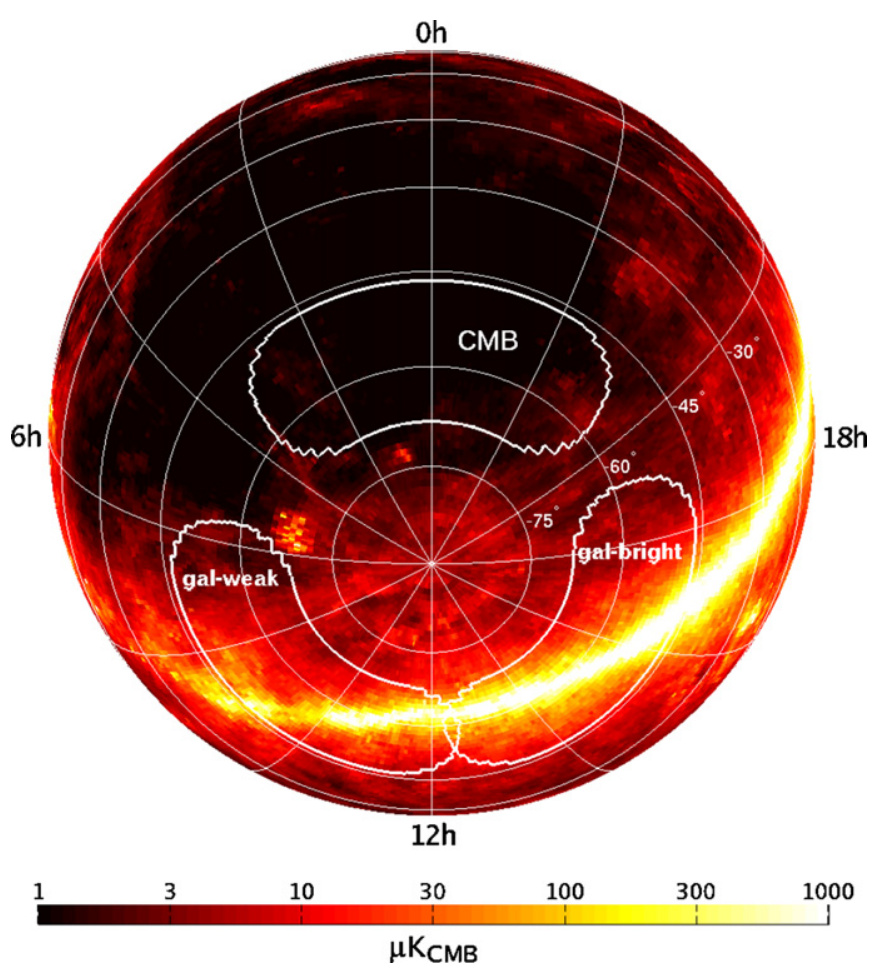

Figure 1. BICEP's CMB and Galactic fields are outlined on the $150 \mathrm{GHz}$ FDS Model 8 prediction of dust emission (Finkbeiner et al. 1999), plotted here in equatorial coordinates.

a region of low dust emission outlined in Figure 1, in a right ascension and declination range of approximately $|\alpha|<60^{\circ}$ and $-70^{\circ}<\delta<-45^{\circ}$. The telescope observation cycle is 48 sidereal hours in length and is divided into four $9 \mathrm{hr} \mathrm{CMB}$ observations, $6 \mathrm{hr}$ of Galactic observations, and $6 \mathrm{hr}$ of cryogen servicing. The CMB field is covered twice over the same azimuth range during each $48 \mathrm{hr}$ cycle, but the elevation halves are mapped in opposite order between the two observations. The boresight angle in each cycle is held fixed at one of four angles $\left\{-45^{\circ}, 0^{\circ}, 180^{\circ}, 135^{\circ}\right\}$ that provide good thermal-microphonic stability and redundant polarization angle coverage.

BICEP maps the sky with azimuth-elevation raster scans. During each complete CMB observation (18 hr), the telescope boresight steps in elevation between $55^{\circ}$ and 59.75 in 0.25 increments. At each step in elevation, the telescope performs a set of 50 back-and-forth azimuth scans over a total period of $\sim 50$ minutes. The azimuth scan width is 64.4 , and the speed is held constant at $2.8 \mathrm{~s}^{-1}$ over $\sim 70 \%$ of the scan duration, which modulates the sky signal and places it in a frequency band of approximately $0.1-1 \mathrm{~Hz}$. The scans have a fixed azimuth center that is updated at each elevation step to approximately track the field center. This scan strategy was chosen instead of continuous tracking in order to allow removal of any azimuth-fixed or scansynchronous contamination.

Relative detector gains are measured regularly during observing cycles with "elevation nods" performed at the beginning and end of each fixed-elevation scan set. During an elevation nod, the mount performs a rounded triangle wave motion in elevation with a 1.2 peak-to-peak amplitude, and the detector voltages vary in response to the changing line-of-sight air mass. The nod is performed over a $45 \mathrm{~s}$ period to reduce thermal disturbances on the focal plane, and thermal drifts are further suppressed by using mirror-image elevation nods at the beginning and end of each scan set (up-down-up and down-up-down). 


\section{INSTRUMENT CHARACTERIZATION}

The timestream $d(t)$ of a perfect linearly polarized detector is related to the signal on the sky through the expression $d(t)=T\left(\mathbf{r}_{b}\right)+Q\left(\mathbf{r}_{b}\right) \cos 2 \psi+U\left(\mathbf{r}_{b}\right) \sin 2 \psi$, where the sky signal is described by the Stokes parameters $T, Q, U$. This expression depends only on the detector's direction of observation $\mathbf{r}_{b}$ and polarization orientation angle $\psi$. (The time-dependence of $\mathbf{r}_{b}$ and $\psi$ that arises from the scan strategy is suppressed for clarity.) Modifying this simple expression, the timestream response of a BICEP PSB is described by

$$
\begin{aligned}
d(t) & =K(t) *\left\{n(t)+g(t) \int d v A_{e} F(v) \int d \mathbf{r} P\left(\mathbf{r}-\mathbf{r}_{b}, v\right)\right. \\
\times & {\left.\left[T(\mathbf{r}, v)+\frac{1-\epsilon}{1+\epsilon}(Q(\mathbf{r}, v) \cos 2 \psi+U(\mathbf{r}, v) \sin 2 \psi)\right]\right\}, }
\end{aligned}
$$

where the extra terms are calibration quantities that describe the properties of the instrument. The cross-polar leakage, which is a PSB's level of response to orthogonally polarized light, is parameterized by $\epsilon$. The detector signal is convolved with the co-polar beam $P(\mathbf{r}, v)$, which has a spatial extent that depends on the coordinate $\mathbf{r}$. The beam-convolved detector response is integrated over the pass band $F(v)$, which is multiplied by the effective antenna area $A_{e}$. The gain factor $g(t)$ converts voltage to temperature units, and $n(t)$ is an additive noise term. Finally, the entire expression is convolved with the detector transfer function $K(t)$.

In order to faithfully reconstruct the temperature and polarization signal on the sky, it is necessary to measure all the terms in Equation (1) that relate $T, Q, U$ to the detector voltage. A complete description of these measurement procedures and results is given in the accompanying instrument characterization paper (Takahashi et al. 2010). Here, we give only a brief summary of the quantities used directly in data analysis. The list includes detector transfer functions, absolute and relative gains, main beam shapes, cross-polar leakages, detector orientation angles, and pointing. The noise is discussed separately in Section 6.1.

\subsection{Transfer Functions}

Deconvolving detector transfer functions is the first step in producing clean timestreams that are suitable for analysis. Relative gains are measured with elevation nods at $0.02 \mathrm{~Hz}$, so the transfer functions must be characterized over a frequency range that spans at least $0.01-1 \mathrm{~Hz}$ in order to link the relative gains to the entire science band. The transfer functions were measured with a microwave source (Gunn oscillator or broadband noise source) that was placed near the telescope window and square-wave modulated at $0.01 \mathrm{~Hz}$. The timedomain responses to the transitions were Fourier transformed, divided by the transform of the modulation waveform, and averaged for each detector in order to obtain the deconvolution kernel. The transfer functions have a measurement precision of $0.5 \%$ rms across the signal band and have sufficiently high signal-to-noise ratio $(\mathrm{S} / \mathrm{N})$ to be deconvolved directly from the bolometer timestreams. (Although the transfer functions can be described by a simple model, we do not rely on those fits.) The relative gain uncertainty that results from transfer function deconvolution is $<0.3 \%$ over the range $0.01-1 \mathrm{~Hz}$.

\subsection{Relative and Absolute Gains}

PSB relative gains are measured with elevation nods at the beginning and end of every set of constant-elevation azimuth scans. The nods inject an atmospheric signal into the bolometer timestreams, and the gains are obtained by fitting each timestream against the cosecant of the detector elevation. The resulting volts-per-air mass responsivity factors are normalized to the average of good detectors for each frequency band during the scan set. The common-mode rejection of CMB temperature anisotropies in gain-adjusted PSB pairs is measured to be better than $98.9 \%$ at degree angular scales.

We cross-correlate the CMB temperature fluctuations measured by WMAP and BICEP to obtain absolute gains, which relate CMB temperature to detector units. The WMAP maps are smoothed to BICEP's resolution by applying the ratio of the beam window functions, $B_{\ell}^{\mathrm{BICEP}} / B_{\ell}^{\mathrm{WMAP}}$. (We do not apply a correction for the pixelization of the WMAP maps since the effects are negligible.) The smoothed maps are then converted to simulated detector timestreams using the boresight pointing data. The timestreams are filtered and converted back into maps, thus creating a "BICEP-observed" version of the WMAP data. The BICEP map and processed WMAP maps, which have compatible beam and filter functions, are cross-correlated in multipole space to obtain the absolute gain

$$
g_{b}=\frac{\sum_{\ell} P_{\ell}^{b}\left\langle a_{\ell m}^{\text {WMAP-1 }} a_{\ell m}^{* \text { BICEP }}\right\rangle}{\sum_{\ell} P_{\ell}^{b}\left\langle a_{\ell m}^{\text {WMAP-1 }} a_{\ell m}^{* \text { WMAP }-2}\right\rangle},
$$

where $P_{\ell}^{b}$ is a top hat binning operator, and $a_{\ell m}$ are the spherical harmonic expansion coefficients. To avoid noise bias, the $a_{\ell m}$ coefficients in the denominator are taken from two different WMAP maps; for this analysis, we have used the Q- and V-band maps from the five-year data release (Hinshaw et al. 2009). The resulting gain calibration, $g_{b}$, is approximately flat over BICEP's $\ell$ range of $21-335$, where the lower bound is set by the timestream filtering, and the upper bound is set by beam uncertainty. The absolute gain used for each of the BICEP frequency bands is a single number taken from the average of $g_{b}$ over six uniform bins spanning a multipole window of $56 \leqslant \ell \leqslant 265$, and the absolute gain uncertainty is derived from the standard deviation of $g_{b}$. To assess the impact of errors in the beam window functions, we have calculated $g_{b}$ using both the Q- and V-band WMAP data in the numerator of Equation (2). The average $g_{b}$ values are consistent within errors, and we take the larger of the two standard deviation values, $2 \%$, as a conservative estimate of the absolute gain uncertainty in temperature units.

\subsection{Boresight and Detector Pointing}

The two components of detector pointing reconstruction are telescope boresight pointing and detector offsets relative to the boresight (focal plane coordinates). Raw boresight pointing timestreams are obtained from encoders located on the three mount axes, and corrections to the raw data are applied from a model describing axis tilts and encoder offsets. The pointing model is established with star observations from an optical camera located on the upper surface of the cryostat.

The focal plane coordinates of each detector are reconstructed from measurements of CMB temperature fluctuations. Temperature maps from each detector at each of the four boresight angles are cross-correlated with the full-season map, and the process is repeated with varying centroid adjustments until the maps 
are self-consistent. This method results in $0.03 \mathrm{rms}$ centroid uncertainty.

\subsection{Polarization Orientation and Efficiency}

To recover polarization information from detector timestreams, it is necessary to know the polarization orientation angle $\psi$ and cross-polar leakage $\epsilon$ of each PSB. Note that $\epsilon$ is a property of the detector itself and is independent of the cross-polar beam, which is a property of the optical chain upstream of the PSB. For BICEP, $\epsilon$ dominates over the cross-polar beam. Both $\psi$ and $\epsilon$ are measured with several different devices (e.g., rotating wire grid, dielectric sheet calibrator) that send polarized light into BICEP at many different angles with respect to the detectors. The phase of each PSB's sinusoidal response and the ratio of the minimum to maximum determine $\psi$ and $\epsilon$, respectively. The uncertainty in the measured orientation angles is \pm 0.7 . The median $\epsilon$ for BICEP PSBs is about 0.04 , with a measurement uncertainty of \pm 0.01 .

\subsection{Main Beam Shapes}

The BICEP beams were mapped by raster scanning the telescope over a bright source at various fixed boresight angles. The beams are well described by a Gaussian model, with fit residuals typically about $1 \%$ with respect to the beam amplitude. The beams at both frequencies are nearly circular, with ellipticities under $1.5 \%$. We therefore approximate the beams as symmetric Gaussians with average full widths of 0.93 and 0.60 at 100 and $150 \mathrm{GHz}$, respectively. The distribution of beam widths varies by $\pm 3 \%$ across the focal plane, and each width is measured to a precision of $\pm 0.5 \%$.

\section{LOW-LEVEL ANALYSIS AND MAPMAKING}

The analysis presented here includes BICEP data from the 2006 and 2007 observing seasons. For these initial results, we restrict the data set to uninterrupted $9 \mathrm{hr} \mathrm{CMB}$ observations taken during February-November. Although there is no evidence for Sun contamination, we exclude data acquired during the Austral summer because of mediocre weather conditions and increased station activities. A significant fraction of 2006 was devoted to calibration measurements and investigation of a different scan strategy, and the nominal data set in this season consists of 124 days. The amount of data increased to 245 days in the 2007 season.

\subsection{Data Cuts}

The data set is further reduced by omitting $9 \mathrm{hr}$ observation phases with extremely poor weather quality. For each phase, the standard deviation of relative gains from elevation nods is calculated for each channel, and the median over the 100 and $150 \mathrm{GHz}$ channels of those standard deviations yields two numbers per phase. An observation phase is cut if either of the median standard deviations is greater than $20 \%$ of the average relative gain. After this weather cut, the data set consists of 117 and 226 days for the 2006 and 2007 seasons.

Of the 49 optically active PSB pairs, several are excluded from each season due to anomalous behavior such as no response, excess noise, poorly behaved or time-dependent transfer functions, and exceptionally poor polarization efficiency. Six experimental pixels containing Faraday rotator modules (2006 season only) and the two $220 \mathrm{GHz}$ pixels (2007 and 2008 seasons) are also excluded from CMB analysis. A total of $19100 \mathrm{GHz}$ and 14
$150 \mathrm{GHz}$ PSB pairs are used for 2006 analysis, and $22100 \mathrm{GHz}$ and $15150 \mathrm{GHz}$ PSB pairs are used for 2007.

\subsection{Low-level Timestream Processing}

The raw output of BICEP consists of voltage timestreams sampled at $50 \mathrm{~Hz}$ for 144 channels comprising 98 light bolometers, 12 dark bolometers, 16 thermistors, 10 resistors, and eight intentionally open channels. The low-level timestream cleaning begins with concatenating and trimming the raw data files for each $9 \mathrm{hr}$ observation phase. Complete transfer functions are deconvolved for the 98 light bolometers, and all timestreams are low pass filtered at $5 \mathrm{~Hz}$ and downsampled to $10 \mathrm{~Hz}$. Relative detector gains are derived from elevation nods, and horizon and celestial boresight coordinates are calculated using the pointing model. "Half-scans," or single sweeps in azimuth, are identified, and the turnarounds are excluded from further analysis. The remaining central portion of the half-scan, which has nearly constant velocity, makes up $\sim 75 \%$ of the total scan duration.

Each detector half-scan is subjected to three data quality checks. First, relative gains derived from elevation nods at the beginning and end of a set of scans are compared, and all of the half-scans in the scan set are excluded if the gains differ by more than 3\%. Second, gain-adjusted PSB pairs are differenced over each half-scan, and the half-scan is rejected if the magnitude of the skew or kurtosis is abnormally high (PSB pair-difference timestreams are expected to be Gaussian white noise). Finally, half-scans that contain cosmic rays and other signal spikes are identified by points that are greater than 7 times the standard deviation of the smoothed timestream. On average, the combined half-scan flagging criteria exclude about $3 \%$ of all half-scans over all light detectors. Because the flagged percentage is small, the problematic half-scans are not gap-filled and are simply omitted from analysis.

\subsection{Mapmaking}

After low-level cleaning, the bolometer timestreams are binned into temperature and polarization maps. We have developed two data analysis pipelines for BICEP that differ starting from the mapmaking stage. The code in each pipeline is completely independent of the other, and the only shared data products are the initial set of downsampled, cleaned detector timestreams, boresight pointing, and calibration data. Both pipelines have reproduced all the results reported in this paper, achieving similar statistical power and excellent agreement. The mapmaking algorithms used by the two pipelines are similar, although one (designated for this paper the "primary pipeline") bins in the Healpix (Górski et al. 2005) pixelization scheme, while the other (designated for this paper as the "alternate pipeline") produces maps in rectangular coordinates. In this section, we describe the primary pipeline's mapmaking procedure in detail.

Following Jones et al. (2007), the simplified timestream output $d_{i j}$ of a single PSB can be expressed as

$$
d_{i j}=g_{i j}\left[T\left(\mathbf{p}_{j}\right)+\gamma_{i}\left(Q\left(\mathbf{p}_{j}\right) \cos 2 \psi_{i j}+U\left(\mathbf{p}_{j}\right) \sin 2 \psi_{i j}\right)\right],
$$

where $g$ is the gain, $T, Q, U$ are the beam-integrated Stokes parameters of the sky signal, $\gamma \equiv(1-\epsilon) /(1+\epsilon)$ is the polarization efficiency factor, and $\psi$ is the PSB polarization orientation projected on the sky. The index $i$ denotes the PSB channel number, $j$ is the timestream sample number, and $\mathbf{p}_{j}$ is the map pixel observed at time $j$. The goal of mapmaking is to recover $T, Q, U$ from the bolometer timestreams. 
The mapmaking procedure for BICEP begins with the formation of gain-adjusted sum and difference timestreams for each PSB pair:

$$
d_{i j}^{ \pm}=\frac{1}{2}\left(d_{2 i, j} / g_{2 i, j} \pm d_{2 i+1, j} / g_{2 i+1, j}\right) .
$$

To reduce atmospheric $1 / f$ noise, a third-order polynomial is subtracted from the sum and difference timestreams for each half-scan in azimuth. Azimuth-fixed and scan-synchronous contamination are removed by subtracting a template signal, which is formed by binning the polynomial-filtered detector timestreams in azimuth over each set of fixed-elevation scans. There are slight differences in the scan-synchronous signal between left- and right-going half-scans, so separate templates are calculated for each case. The scan-synchronous contamination removed in this step is very small; $Q$ and $U$ maps typically change by $100-400 \mathrm{nK}$ at the largest scales after its removal.

The temperature $T$ at each map pixel $\mathbf{p}$ is recovered from the filtered sum timestreams with

$$
\left(\sum_{i}^{\text {No.ofpairs }} \sum_{j \in \mathbf{p}} w_{i j}^{+} d_{i j}^{+}\right) /\left(\sum_{i}^{\text {No.ofpairs }} \sum_{j \in \mathbf{p}} w_{i j}^{+}\right) \simeq T(\mathbf{p}),
$$

where $w^{+}$is the weight assigned to each pair sum, and we have assumed that the effects of polarization leakage are negligible. In other words, the temperature is obtained simply by binning the filtered detector timestreams into map pixels. Stokes $Q$ and $U$ are calculated from linear combinations of the difference timestreams using the matrix equation

$$
\begin{aligned}
& \sum_{i}^{\text {No.ofpairs }} \sum_{j \in \mathbf{p}} w_{i j}^{-}\left(\begin{array}{c}
d_{i j}^{-} \alpha_{i j} \\
d_{i j}^{-} \beta_{i j}
\end{array}\right) \\
& \quad=\frac{1}{2} \sum_{i}^{\text {No.ofpairs }} \sum_{j \in \mathbf{p}} w_{i j}^{-}\left(\begin{array}{cc}
\alpha_{i j}^{2} & \alpha_{i j} \beta_{i j} \\
\alpha_{i j} \beta_{i j} & \beta_{i j}^{2}
\end{array}\right)\left(\begin{array}{l}
Q(\mathbf{p}) \\
U(\mathbf{p})
\end{array}\right) .
\end{aligned}
$$

Here, $w^{-}$is the weight assigned to each pair difference, and $\alpha, \beta$ are PSB pair orientation angle factors defined as

$$
\begin{gathered}
\alpha_{i j}=\gamma_{2 i} \cos 2 \psi_{2 i, j}-\gamma_{2 i+1} \cos 2 \psi_{2 i+1, j}, \\
\beta_{i j}=\gamma_{2 i} \sin 2 \psi_{2 i, j}-\gamma_{2 i+1} \sin 2 \psi_{2 i+1, j} .
\end{gathered}
$$

The $2 \times 2$ matrix in Equation (6) is singular for a single pair of $\psi_{2 i, j}$ and $\psi_{2 i+1, j}$, and the equation can be solved only by accumulating more than one timestream sample in a given map pixel p. As $\mathbf{p}$ is observed with many detector polarization angles $\psi$, the off-diagonal $\alpha_{i j} \beta_{i j}$ terms average to zero, and the matrix becomes invertible. Although only two different polarization angles are required to invert the matrix, some instrumental systematics average down as the number of observation angles increases. By examining the determinant of the matrix, pixels (typically at the edge of the map) with insufficient polarization angle coverage or low integration time are identified and masked from analysis.

We choose the pair sum and difference weights $w^{ \pm}$to be proportional to the inverse variance of the filtered timestreams. The weights are evaluated from power spectral densities averaged over each set of azimuth scans (every 50 minutes), a period during which the noise properties are approximately stationary. For each channel pair, the sum/difference weight for a scan set is calculated from the inverse of the average value of the auto-correlation between 0.5 and $1 \mathrm{~Hz}$.

\section{MAP RESULTS}

Figure 2 shows the BICEP maps of CMB temperature and Stokes $Q$ and $U$ parameters. The pixelization is 0.23 , corresponding to Healpix nside $=256$, and the total observation area covers $\sim 2 \%$ of the sky. In the central part of the maps, the integration time exceeds $6 \times 10^{5}$ detector-seconds per square degree, and the scan strategy smoothly apodizes the outer edges of the observed region. The temperature anisotropies are measured with high $\mathrm{S} / \mathrm{N}$ and agree well between the two frequencies. The rms noise per square degree, obtained from simulations, is $0.81 \mu \mathrm{K}$ and $0.64 \mu \mathrm{K}$ for $100 \mathrm{GHz}$ and $150 \mathrm{GHz}$, respectively, in the central region of the $Q$ and $U$ maps.

Data from both frequency bands are combined to form the temperature, $E$, and $B$ maps shown in Figure 3. The maps from each frequency band are smoothed to $1^{\circ}$ resolution, and the left column shows the sum. Frequency jackknife maps that are formed by differencing the 100 and $150 \mathrm{GHz}$ data are shown in the right column. The high $\mathrm{S} / \mathrm{N}$ of the temperature measurement is demonstrated by the lack of structure in the frequency jackknife map. The faint striping, which is caused by residual unpolarized atmospheric noise, is successfully removed by PSB differencing. To form the $E$ and $B$ maps, the $a_{\ell m}^{E}$ and $a_{\ell m}^{B}$ coefficients are computed from apodized $Q$ and $U$ maps using the anafast utility in the Healpix code package. The coefficients are then boxcar filtered in a multipole range of $70<\ell<280$. Using the synfast utility, $a_{\ell m}^{E, B}$ are interpreted as $Y_{\ell m}$ coefficients from which the $E$ and $B$ maps are generated. The $E$ frequency-sum map shows resolved degreescale structure of the expected amplitude, while the $E$ and $B$ jackknife maps, as well as the $B$ signal map, are consistent with noise. These $E$ and $B$ maps are used only as a qualitative illustration of the $\mathrm{S} / \mathrm{N}$ of the data. Since the method of creating the maps has several shortcomings, e.g., there is some $E / B$ mixing due to the finite survey area, we do not use the $E$ and $B$ maps in any quantitative analysis of the power spectra.

\section{POWER SPECTRUM ESTIMATION}

BICEP employs two different analysis pipelines for power spectrum estimation: the first performs a curved-sky analysis using the publicly available Spice (Chon et al. 2004) package to estimate the power spectra, and the second is a flat-sky pseudo$C_{\ell}$ estimator adapted from the QUAD pipeline that was used in the Pryke et al. (2009) main analysis. In the following, we describe in detail the Spice pipeline.

The power spectra are derived from inverse variance weighted $T, Q$, and $U$ maps. Temperature and polarization correlation functions are computed from fast spherical harmonic transforms of the heuristically weighted maps. The polarization correlation functions are then decoupled so that $E / B$ mixing that is caused by the finite survey area is removed in the mean. The correlation functions are apodized in order to reduce correlations in multipole space that result from incomplete sky coverage. Finally, estimates of the full sky power spectra are computed from integral transforms of the apodized correlation functions. The observed power spectra, as computed by Spice, are approximated as

$$
\hat{C}_{\ell}^{X}=\sum_{\ell^{\prime}} \kappa_{\ell \ell^{\prime}}^{X} F_{\ell^{\prime}}^{X} B_{\ell^{\prime}}^{2} C_{\ell^{\prime}}^{X}+\hat{N}_{\ell}^{X},
$$

where the superscripts, $X$, correspond to the six temperaturepolarization combinations $\{T T, E E, B B, T E, T B, E B\}$. The convolution kernel, $\kappa_{\ell \ell^{\prime}}^{X}$, is the normalized Spice window 


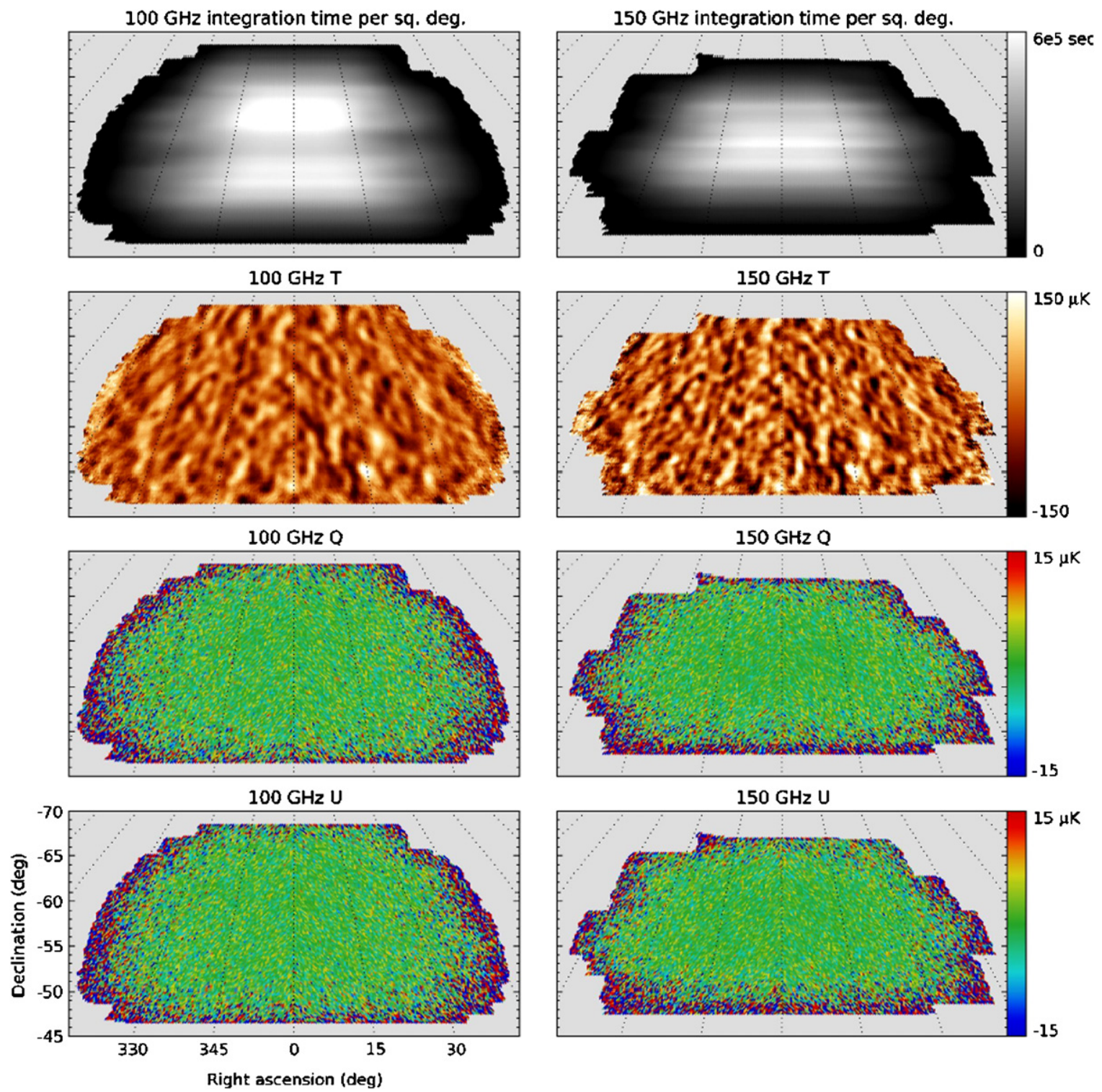

Figure 2. BicEP $T, Q, U$, and coverage maps. The resolution is about 0.9 and 0.6 at 100 and $150 \mathrm{GHz}$, respectively, and no smoothing or apodizing has been applied to the maps. The noise per square degree in the central region of the $Q$ and $U$ maps is $0.81 \mu \mathrm{K}$ at $100 \mathrm{GHz}$ and $0.64 \mu \mathrm{K}$ at $150 \mathrm{GHz}$. Note that the color scales of the temperature and polarization maps differ by a factor of 10 .

function and depends on the apodization function applied to the correlation functions. The effect of timestream filtering is described by $F_{\ell}^{X}$, the $\ell$-space transfer function; $B_{\ell}^{2}=B_{\ell}^{2} H_{\ell}^{2}$ is the product of the beam and pixel window functions, and $\hat{N}_{\ell}^{X}$ is the power spectrum of the noise convolved with the Spice kernel. In the following subsections, we describe the steps in solving Equation (9) and recovering an estimate of the underlying power spectrum, $C_{\ell}^{X}$.

\subsection{Noise Subtraction}

The first step in power spectrum estimation is calculating and subtracting the noise bias $\hat{N}_{\ell}$. We estimate $\hat{N}_{\ell}$ with Monte Carlo simulations of instrument noise: starting from a noise model, we generate simulated timestreams that are filtered, co-added into maps, and processed with Spice. The resulting simulated noise spectra are averaged over many realizations to form $\hat{N}_{\ell}$.

The BICEP noise model is derived from gain-adjusted PSB pair sum and difference timestreams (Equation (4)) under the assumption that the timestream $\mathrm{S} / \mathrm{N}$ is negligible, i.e., the signal is the noise. The $\mathrm{S} / \mathrm{N}$ is $\leqslant 10 \%$ and $\leqslant 0.1 \%$ for the sum and difference timestreams, respectively. The sum and difference timestreams are polynomial-filtered and Fourier transformed over each half-scan, and all auto-correlations and cross-correlations between channel pairs are computed to form the complex frequency-domain noise covariance matrix $\tilde{N}(f)$. To form the noise model, we average $\tilde{N}(f)$ over the 100 half-scans within each scan set and then average into 12 logarithmically spaced bins spanning $0.05-5 \mathrm{~Hz}$.

To construct simulated correlated noise timestreams, we take the Cholesky decomposition $\tilde{N}(f)=\mathbf{L}(f) \mathbf{L}^{\dagger}(f)$ of the noise covariance matrix and multiply a vector of pseudo-random complex numbers $\boldsymbol{\rho}(f)$ by $\mathbf{L}(f)$ :

$$
\tilde{\boldsymbol{v}}(f)=\mathbf{L}(f) \boldsymbol{\rho}(f) .
$$

The complex numbers in $\rho(f)$ have Gaussian-distributed real and imaginary components and are normalized such that the magnitude has a standard deviation of one. The resulting product 
$\mathrm{T}(100+150 \mathrm{GHz}) / 2$

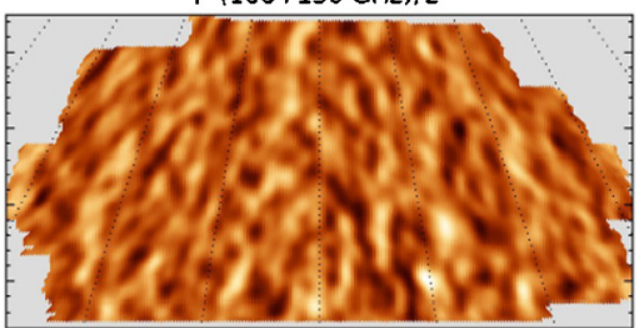

$E(100+150 \mathrm{GHz}) / 2$

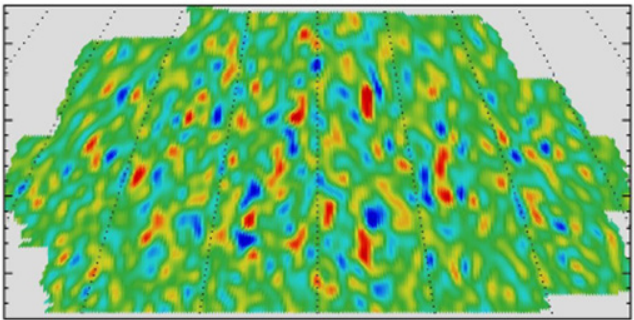

B $(100+150 \mathrm{GHz}) / 2$

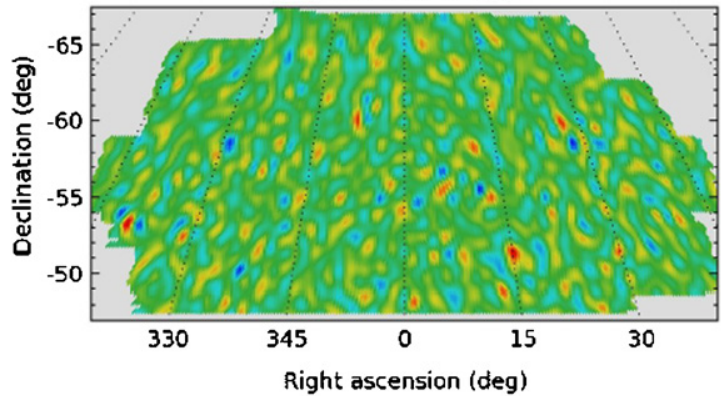

T Jackknife $(100-150 \mathrm{GHz}) / 2$

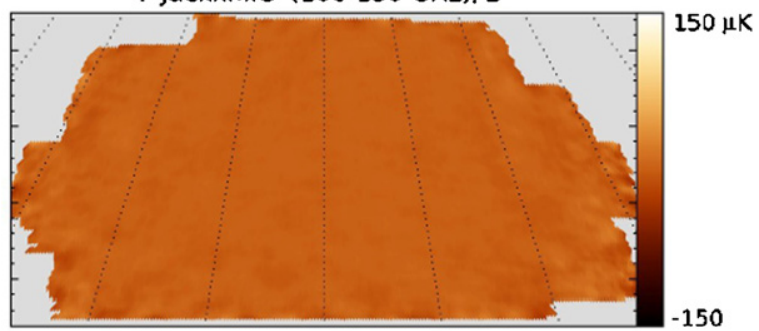

E Jackknife $(100-150 \mathrm{GHz}) / 2$

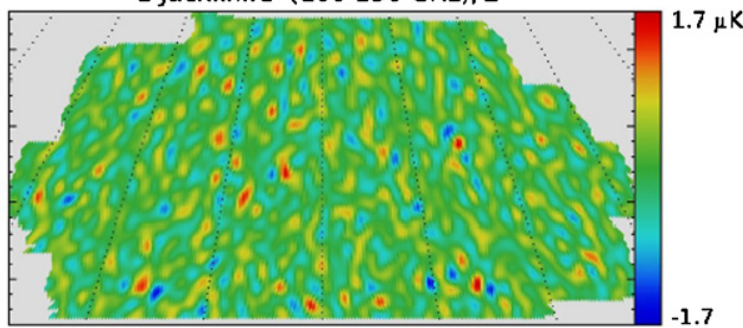

$-1.7$

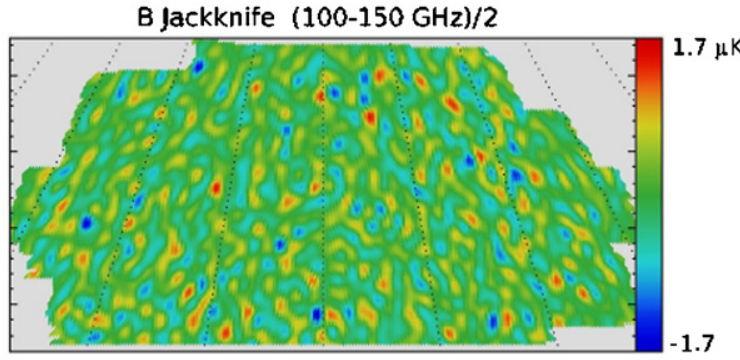

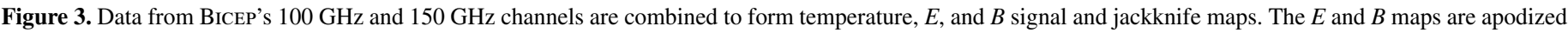

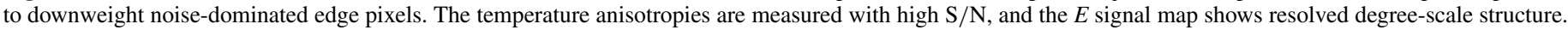
The $B$ signal map and the $E$ and $B$ jackknife maps are consistent with noise.

$\tilde{\boldsymbol{v}}(f)$ has the same statistical properties as the data and is inverse Fourier transformed to obtain a set of simulated noise timestreams. Scan-synchronous templates are calculated and subtracted from each set of azimuth scans, and the filtered noise timestreams are then co-added into maps.

The noise bias, $\hat{N}_{\ell}$, is estimated by averaging the power spectra from an ensemble of noise-only maps. Figure 4 shows $\hat{N}_{\ell}$ (red dashed lines), calculated from the average over 500 realizations, in comparison to raw power spectra. BICEP measures $T T$ and $T E$ with high $\mathrm{S} / \mathrm{N}$, and the noise contribution is negligible up to $\ell \sim 330$. The noise is mostly uncorrelated between temperature and polarization, so $\hat{N}_{\ell}$ for $T B$ is distributed around zero, and the same is true for $E B$. In contrast, noise comprises the bulk of the $E E$ and all of the $B B$ amplitude at $\ell>150$, which illustrates the need for careful noise modeling and subtraction. We have studied the impact of noise misestimation by intentionally scaling the noise spectra (Takahashi et al. 2010), and we find that the largest possible error in $\hat{N}_{\ell}$ is $\pm 3 \%$.

\subsection{Beam and Pixelization Corrections}

The finite resolution of the telescope and pixelization of the maps result in suppression of the observed power spectra at small angular scales. This suppression of power is described by $B_{\ell}^{2}=B_{\ell}^{2} H_{\ell}^{2}$, the product of the beam and pixel window functions, and is illustrated in Figure 5. In this analysis, we approximate $B_{\ell}$ as the Legendre transform of the average beam within a given frequency band, which is assumed to be a circular Gaussian. The full widths at 100 and $150 \mathrm{GHz}$ are 0.93 and
0.60 , respectively. The pixel window function $H_{\ell}$ is supplied by Healpix and corresponds to nside $=256$. In the case of BICEP, $B_{\ell}$ varies slowly with respect to the Spice kernel, $\kappa_{\ell \ell^{\prime}}^{X}$, and can therefore be pulled out of the convolution. The observed power spectra, after noise subtraction, are divided by $B_{\ell}^{2}$ to correct for the effects of beam suppression and pixelization:

$$
\left(\hat{C}_{\ell}^{X}-\hat{N}_{\ell}^{X}\right) / B_{\ell}^{2} \simeq \sum_{\ell^{\prime}} \kappa_{\ell \ell^{\prime}}^{X} F_{\ell^{\prime}}^{X} C_{\ell^{\prime}}^{X}
$$

\subsection{Filter Corrections}

The bolometer timestreams are cleaned by subtracting a thirdorder polynomial and scan-synchronous template; this cleaning procedure also has the effect of removing large-scale CMB signal. The amount of signal loss is described by $F_{\ell}$, the $\ell$-space transfer function imposed by timestream filtering. In addition, although the Spice estimator is unbiased in the mean, the timestream processing removes spatial modes from the observed $T, Q$, and $U$ maps, generically introducing couplings between the observed $E$ and $B$ spectra. These couplings are small, but are of interest for the leakage of the relatively large $E$-mode signal into $B$-mode polarization. We characterize both $F_{\ell}$ and $E$-to- $B$ leakage with signal-only simulations.

The signal simulation procedure begins by generating model power spectra using the CAMB (Lewis et al. 2000) software package. We use $\Lambda \mathrm{CDM}$ parameters derived from WMAP fiveyear data (Hinshaw et al. 2009) and a tensor-to-scalar ratio of zero. From the model spectra, we use the synfast utility 

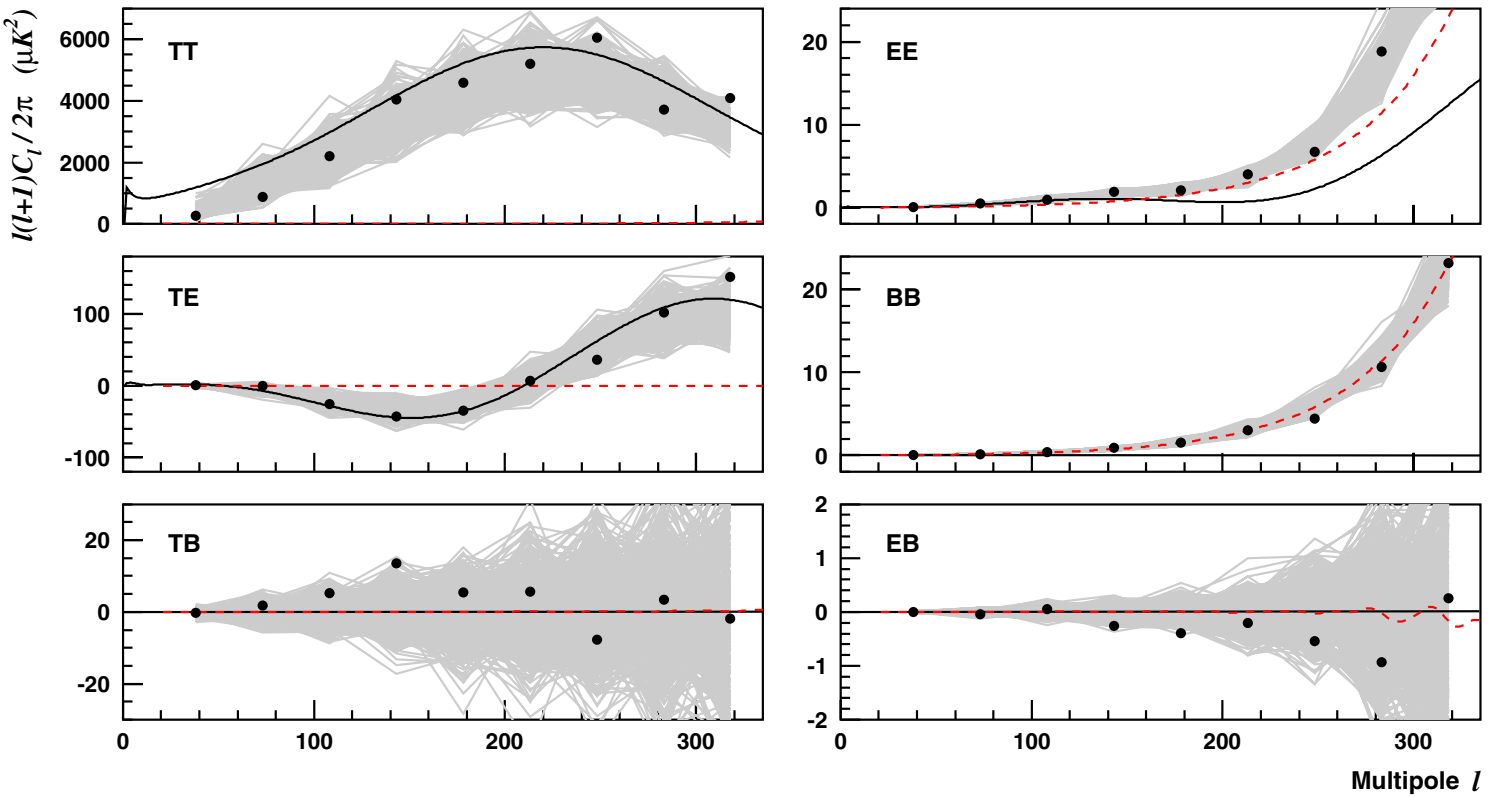

Figure 4. Raw power spectra, uncorrected for noise and filter bias, are shown here for 500 signal-plus-noise simulations (gray lines) and BICEP 150 GHz data (black points). The dashed red lines indicate the level of noise bias, $\ell(\ell+1) \hat{N}_{\ell} /(2 \pi)$, and the black lines show the $\Lambda$ CDM spectra used as inputs for the signal simulations. Noise dominates much of the $E E$ spectrum and all of the $B B$ spectrum. The effect of filter bias is visible in the low- $\ell$ portion of the $T T$ spectrum, where the simulated spectra fall below the model curve.

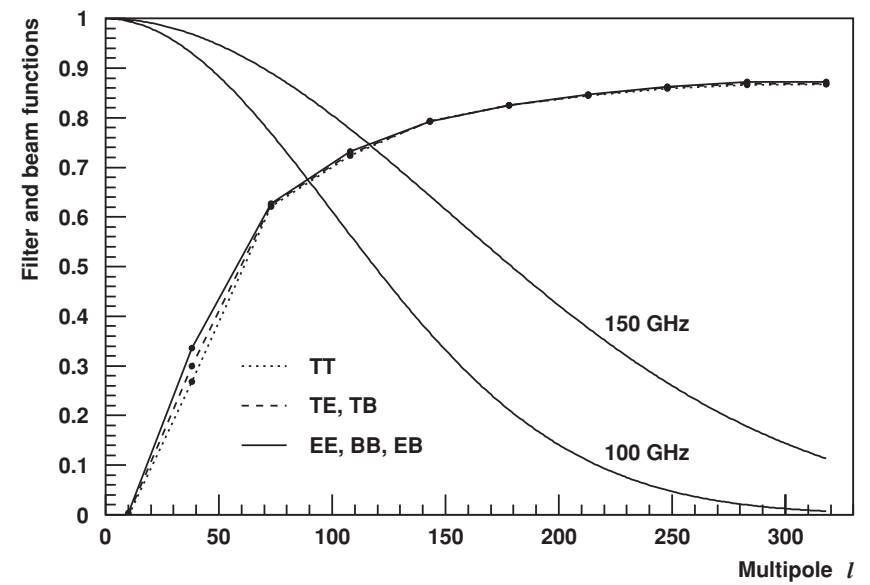

Figure 5. Signal-only simulations are used to evaluate the filter function $F_{b}^{X}$, shown here for $150 \mathrm{GHz}$ ( $F_{b}^{X}$ for other frequency combinations look similar). The beam-pixel window functions $B_{\ell}^{2}=B_{\ell}^{2} H_{\ell}^{2}$ are shown for 100 and $150 \mathrm{GHz}$.

in Healpix to create an ensemble of simulated CMB skies pixelized at 0.11 resolution $($ nside $=512)$ and convolved with the BICEP beams. Actual pointing data are used to generate smoothly interpolated PSB timestreams from the simulated $T, Q, U$ maps and their derivatives. A PSB timestream sample $d(\mathbf{p})$ that falls into a pixel centered at $\mathbf{p}$ is expressed as a convolution of the beam $P\left(\mathbf{r}-\mathbf{r}_{b}\right)$, which is centered at $\mathbf{r}_{b}$, with a second-order Taylor expansion of the sky signal $m$ around $\mathbf{p}$ (E. F. Hivon \& N. Ponthieu 2010, in preparation):

$$
\begin{aligned}
d(\mathbf{p})= & \int d \mathbf{r} P\left(\mathbf{r}-\mathbf{r}_{b}\right)[m(\mathbf{p})+\vec{\nabla} m(\mathbf{p})(\mathbf{r}-\mathbf{p}) \\
& \left.+\frac{1}{2}(\mathbf{r}-\mathbf{p})^{T} D^{2} m(\mathbf{p})(\mathbf{r}-\mathbf{p})\right] .
\end{aligned}
$$

Here, $m(\mathbf{p})=g[T(\mathbf{p})+\gamma(Q(\mathbf{p}) \cos 2 \psi+U(\mathbf{p}) \sin 2 \psi)]$, and $\vec{\nabla}$ and $D^{2}$ denote the first and second derivatives in spherical coordinates. Assuming Gaussian beams for each frequency band, Equation (12) reduces to

$$
\begin{aligned}
d(\mathbf{p})= & m(\mathbf{p})+\vec{\nabla} m(\mathbf{p})\left(\mathbf{r}_{b}-\mathbf{p}\right) \\
& +\frac{1}{2} \operatorname{Tr}\left[D^{2} m(\mathbf{p})\left(\begin{array}{cc}
(\Delta \phi)^{2} & \Delta \phi \Delta \theta \\
\Delta \phi \Delta \theta & (\Delta \theta)^{2}
\end{array}\right)\right],
\end{aligned}
$$

where $\Delta \phi$ and $\Delta \theta$ are the longitude and latitude offsets between the pointing vector $\mathbf{r}_{b}$ and the pixel center $\mathbf{p}$. We apply Equation (13) to simulate signal-only detector timestreams according to BICEP's scan strategy. Measured PSB pair centroids, detector orientation angles, and cross-polar leakage values are included in the simulations. For the purpose of characterizing the effects of timestream filtering, all differential beam systematic effects are turned off so that there is no mixing between temperature and polarization. The simulated timestreams are filtered and weighted in exactly the same way as the real data and then co-added into maps.

Once we have "BICEP-observed" signal-only maps in hand, we compute the power spectra with Spice and average the results over many realizations. Because the input CAMB spectra have $r=0$, any non-zero $B B$ power in the simulation outputs is interpreted as contamination from $E$-mode polarization that is induced by timestream filtering. We therefore apply a correction,

$$
\left(\hat{C}_{\ell}^{B B}-\hat{N}_{\ell}^{B B}-\left.\hat{C}_{\ell}^{B B}\right|_{E E \text { only }}\right) / B_{\ell}^{2} \simeq \sum_{\ell^{\prime}} \kappa_{\ell \ell^{\prime}}^{B B} F_{\ell^{\prime}}^{B B} C_{\ell^{\prime}}^{B B},
$$

to the $B B$ power spectrum of the data, where $\left.\hat{C}_{\ell}^{B B}\right|_{E E \text { only }}$ is the ensemble average $B B$ spectrum from the $r=0$ signal simulation outputs. The amplitude of this correction is roughly $\ell(\ell+$ 1) $\left.\hat{C}_{\ell}^{B B}\right|_{E E \text { only }} /(2 \pi) \simeq 3 \times 10^{-3} \mu \mathrm{K}^{2}$ at $\ell \sim 100$, comparable to inflationary $B B$ power for $r=0.05$, and the sample variance is about $3 \times 10^{-3} \mu \mathrm{K}^{2}$. The correction factors for the other spectra are negligibly small, so only the $B B$ spectrum is adjusted with this procedure. 
To quantify the suppression of large-scale power from timestream filtering, we begin by assuming that $F_{\ell}^{X}$, like $B_{\ell}^{2}$, varies slowly in comparison to the Spice kernel and can be pulled out of the convolution. We choose to calculate the binned filter suppression factor, $F_{b}^{X}$, defined as

$$
\sum_{\ell} P_{\ell}^{b} \frac{\ell(\ell+1)}{2 \pi} \frac{\left(\hat{C}_{\ell}^{X}-\hat{N}_{\ell}^{X}\right)}{B_{\ell}^{2}} \simeq F_{b}^{X} \sum_{\ell} P_{\ell}^{b} \frac{\ell(\ell+1)}{2 \pi} \sum_{\ell^{\prime}} \kappa_{\ell \ell^{\prime}} C_{\ell^{\prime}}^{X},
$$

where the binning operator $P_{\ell}^{b}$ is a top hat. (In the case of the $B B$ spectrum, the left hand side of the equation also contains $\left.\hat{C}_{\ell}^{B B}\right|_{E E \text { only }}$, as in Equation (14).) We obtain the filter suppression factors by comparing the power spectra of the BICEP-observed signal-only maps to those of the input synf ast maps; $F_{b}^{X}$ is the ratio of the spectra, after multiplying by $\ell(\ell+1) /(2 \pi)$ and binning.

Figure 5 shows $F_{b}^{X}$ averaged over 500 signal simulations using BICEP's timestream filtering. In each simulation, signalonly timestreams are generated from the full two years of pointing data. At $\ell \sim 100$, the value of $F_{b}^{X}$ is about 0.7 for all spectra and rises slowly as $\ell$ increases. Identical filter functions are used for the $E E$ and $B B$ spectra, and the filter functions of the cross-spectra are calculated as the geometric mean of those determined from the auto-spectra. The validity of this approach for obtaining the $B B$ and cross-spectra filter functions has been confirmed with simulations over a limited multipole range. Dividing by $F_{b}^{X}$ is the final step in obtaining the BICEP band power estimates:

$$
\begin{gathered}
\hat{\mathscr{C}}_{b}^{X} \equiv \frac{1}{F_{b}^{X}} \sum_{\ell} P_{\ell}^{b} \frac{\ell(\ell+1)}{2 \pi} \frac{\left(\hat{C}_{\ell}^{X}-\hat{N}_{\ell}^{X}\right)}{B_{\ell}^{2}}, X \neq B B \\
\hat{\mathscr{C}}_{b}^{B B} \equiv \frac{1}{F_{b}^{B B}} \sum_{\ell} P_{\ell}^{b} \frac{\ell(\ell+1)}{2 \pi} \frac{\left(\hat{C}_{\ell}^{B B}-\hat{N}_{\ell}^{B B}-\left.\hat{C}_{\ell}^{B B}\right|_{E E \text { only }}\right)}{B_{\ell}^{2}} .
\end{gathered}
$$

\subsection{Error Bars}

The uncertainties in the power spectra consist of two components, one that is proportional to the signal itself (sample variance), and another that depends on the instrumental noise. We estimate the errors by examining the variance of power spectra from simulated signal-plus-noise maps, which exactly encode time-dependent correlated noise, scan strategy, and sky coverage. We add the simulated noise-only and signal-only maps, described in Sections 6.1 and 6.3; and power spectra are calculated for each realization using the same $\hat{N}_{\ell}, B_{\ell}^{2}$, and $F_{b}^{X}$ as applied to the real data. If the simulations include a reasonable model of the signal and faithfully reproduce all the noise properties of the experiment, then the data and simulations should be indistinguishable. Figure 4 shows raw power spectra, uncorrected for noise and filter bias, from 500 signal-plus-noise simulations at $150 \mathrm{GHz}$. The raw spectra of the actual data, shown by the black points, lie within the scatter of the simulations. We calculate the band power covariance matrix from the ensemble of simulations, after applying noise, filter, and beam corrections. The band power errors are obtained from square root of the diagonal terms of the matrix.

\subsection{Band Power Window Functions}

In order to compare our band power estimates to a theoretical model, we need a method to calculate expected band power values from theoretical power spectra. The relationship between the model and the expected band powers is described by band power window functions, $\omega_{\ell}^{b}$, defined as

$$
\mathscr{C}_{b}=\sum_{\ell} \frac{\left(\ell+\frac{1}{2}\right)}{\ell(\ell+1)} \omega_{\ell}^{b} \mathscr{C}_{\ell},
$$

where $\mathscr{C}_{\ell} \equiv \ell(\ell+1) C_{\ell} /(2 \pi)$. The window functions are given by

$$
\omega_{\ell}^{b}=\frac{2}{2 \ell+1} \sum_{\ell^{\prime}} P_{\ell^{\prime}}^{b} \ell^{\prime}\left(\ell^{\prime}+1\right) \kappa_{\ell^{\prime} \ell}
$$

and are normalized such that

$$
\sum_{\ell} \frac{\left(\ell+\frac{1}{2}\right)}{\ell(\ell+1)} \omega_{\ell}^{b}=1
$$

The window functions depend on the Spice kernel, which depends on the apodization function applied to the correlation functions. For this analysis, we apodize the correlation functions with a cosine window that spans $50^{\circ}$. We choose to use a uniform binning of width $\Delta \ell=35$, spanning a multipole range of $21 \leqslant \ell \leqslant 335$. This choice of bin width provides minimally correlated band powers while preserving the spectral resolution determined by the width of the Spice kernel. Figure 6 illustrates BICEP's band power window functions for the nine $\ell$ bins.

Because the $B B$ power spectrum is debiased with the procedure described in Equation (14), we set the $E E$-to- $B B$ window functions to zero. This is a valid approximation as long as the $E E$ spectrum of the signal under consideration is statistically consistent with the measured BICEP band powers (which, as shown in Section 12, are well described by a concordance $\Lambda$ CDM cosmology).

\section{POWER SPECTRUM RESULTS}

Figure 7 shows the full set of BICEP spectra plotted with a $\Lambda C D M$ model derived from WMAP five-year data. The 100 and $150 \mathrm{GHz}$ auto-spectra are shown, as well as the $100 \times 150$ crossspectra. In the case of the $T E, T B$, and $E B$ spectra, we also show the $150 \times 100$ cross-correlation. For each spectrum, we present nine band powers with a uniform bin width of $\Delta \ell=35$, spanning $21 \leqslant \ell \leqslant 335$. The $T T, T E$, and $E E$ spectra are detected with high significance and are already sample-variance limited, and there is no detection of signal in $B B, T B$, and $E B$. The results from BICEP's two analysis pipelines agree well with each other, and Figure 11 shows a comparison of the frequency-combined power spectra (Section 11).

As a cross-check, we have also derived $T T, T E$, and $T B$ spectra using WMAP five-year temperature data in BICEP's CMB field (open circles in Figure 7). The WMAP temperature maps are smoothed and filtered identically to BICEP, as described in Section 3.2. The $T T$ points are calculated from the crosscorrelation of the WMAP Q- and V-band maps, and the TE and $T B$ spectra are calculated using WMAP V-band temperature data and BICEP polarization data. For this comparison, we do not subtract noise bias and instead rely on the fact that the pairs of maps have uncorrelated noise. We also do not attempt to assign error bars. Qualitatively, the spectra formed using WMAP temperature data agree well with the spectra from BICEP temperature and polarization data. Both the BICEP and WMAP temperature maps are strongly signal-dominated; apparently the differences between them, including the noise as well as potential systematics, are at a level that has little impact on the $T T, T E$, or $T B$ power spectra results. 

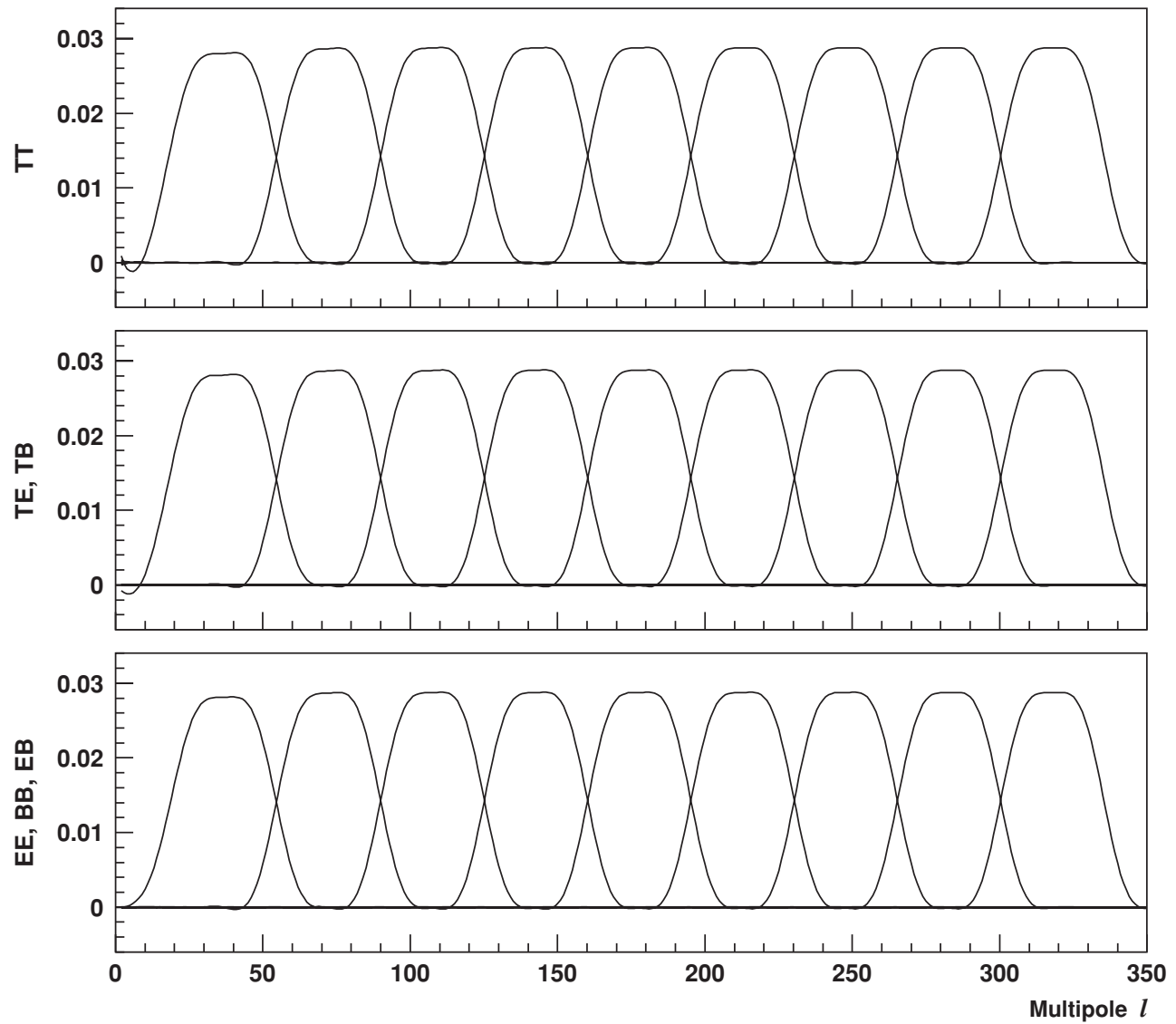

Figure 6. BICEP's band power window functions are defined by Equation (18), and this figure shows $\omega_{\ell}^{b} / \ell$.

\section{CONSISTENCY TESTS}

\subsection{Jackknife Descriptions}

We check the self-consistency of the power spectra by performing jackknives, statistical tests in which the data are split in two halves and differenced. The split is performed at the mapmaking stage, and the resulting differenced map should have power spectra that are consistent with the expected residual signal level (nearly zero) after subtracting noise bias. The interaction of timestream filtering with the details of the split causes imperfect signal cancellation when forming jackknife maps, but in practice, this residual signal is small.

The data are tested with six jackknives that are sensitive to different aspects of the instrument's performance. In the scan direction jackknife, the data are split into left- and right-going azimuth half-scans. Failures generally point to a problem in the detector transfer function deconvolution, or thermal instabilities created at the scan endpoints. The elevation coverage jackknife is formed from the two CMB observations in each $48 \mathrm{hr}$ cycle; each observation covers the same azimuth range but starts from a different elevation. This jackknife is sensitive to groundfixed or scan-synchronous contamination. BICEP observes at four fixed boresight orientation angles that can be split into two pairs, $\left\{-45^{\circ}, 0^{\circ}\right\}$ and $\left\{135^{\circ}, 180^{\circ}\right\}$, to form a boresight angle pair jackknife. This test is perhaps the most powerful of the jackknives performed and is sensitive to many factors, including thermal stability, atmospheric opacity, relative gain mismatches, differential beam pointing, and ground pickup. In the temporal jackknife, the 8 day observing cycles- $48 \mathrm{hr}$ at each of the four boresight angles-are interleaved to form the two halves, and this jackknife tests sensitivity to weather changes. The season split jackknife simply divides the data into the two observing seasons, and failures reflect any changes made to the instrument between the two years. In particular, the focal plane thermal architecture was improved for the 2007 season, and the temperature control scheme was changed. The focal plane $Q U$ jackknife splits the detectors into two groups according to their polarization orientation within the focal plane (approximately alternating hextants) and is a method of probing instrumental polarization effects.

Power spectra of jackknife maps are computed with the method described in Section 6, using simulated jackknife noise and signal-plus-noise maps to subtract noise bias and assign error bars. The filter function $F_{b}^{X}$ is applied so that the magnitude of any non-zero jackknife band powers can be compared to the amplitude of the non-jackknife spectra. For each jackknife spectrum, we calculate $\chi^{2}$ over nine bins spanning $21 \leqslant \ell \leqslant 335$. To account for the expected level of residual signal, the $\chi^{2}$ values are evaluated with respect to the average jackknife spectra from an ensemble of signal-only simulations. The criteria for jackknife success or failure are based on the probability to exceed (PTE) the $\chi^{2}$ value, which is calculated from the distribution of $\chi^{2}$ in 500 signal-plusnoise simulations. Jackknife victory is declared when (1) none of the PTEs are abnormally high or low, given the number of $\chi^{2}$ tests performed, and (2) the PTEs are consistent with a uniform distribution between zero and one.

\subsection{Jackknife Results}

The jackknife spectra for the six different data splits appear similar in that the band powers are all distributed around 
No. 2, 2010
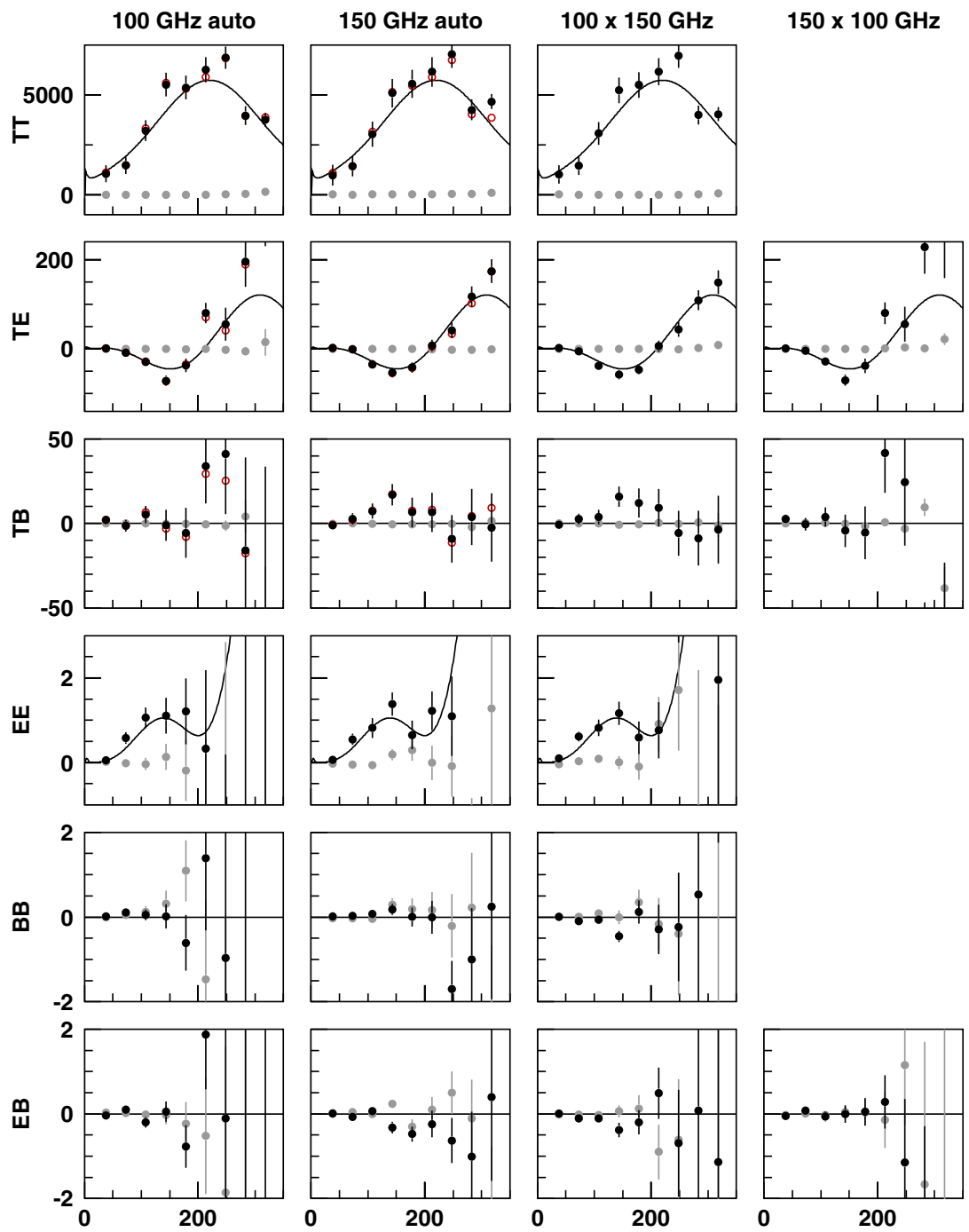

Figure 7. Black points show the full set of BICEP's power spectra. The horizontal axis is multipole moment $\ell$, and the vertical axis is $\ell(\ell+1) C_{\ell} /(2 \pi)$ in units of $\mu \mathrm{K}^{2}$. The spectra agree well with a $\Lambda$ CDM model (black lines) derived from WMAP five-year data and $r=0$. The gray points correspond to the boresight angle pair jackknife; note that although the $T T, T E$, and $T B$ jackknife failures are statistically significant, the amplitudes are small compared to the signal. The open circles show spectra calculated using WMAP five-year temperature data, smoothed and filtered identically to BICEP. We have cross-correlated the WMAP Q- and V-band data in BICEP's CMB field to obtain the TT points, and the $T E$ and $T B$ spectra are calculated from the cross-correlation of WMAP V-band temperature data with BICEP polarization data.

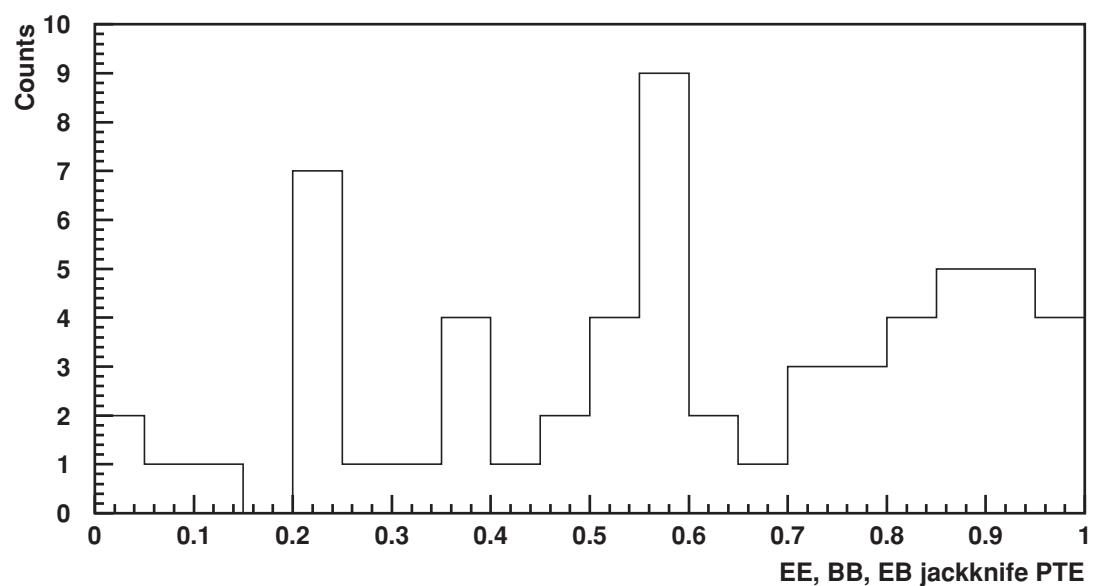

Figure 8. Probabilities to exceed the $\chi^{2}$ values from $E E, B B$, and $E B$ jackknife tests are consistent with a uniform distribution between zero and one.

zero. We therefore show only the spectra from the boresight pair jackknife (Figure 7, gray points), which is arguably the most stringent of the six tests. Table 1 lists the PTE values from all of the jackknife $\chi^{2}$ tests for BICEP's polarization-only spectra $(E E, B B, E B)$. Out of all the PTEs, the lowest value is 0.014 , in the $150 \mathrm{GHz}$ focal plane $Q U$ jackknife. This low 
Table 1

Jackknife PTE Values from $\chi^{2}$ Tests

\begin{tabular}{|c|c|c|c|c|}
\hline Jackknife & $100 \mathrm{GHz}$ & $150 \mathrm{GHz}$ & $100 \times 150$ & $150 \times 100$ \\
\hline \multicolumn{5}{|l|}{ Scan direction } \\
\hline$E E$ & 0.532 & 0.588 & 0.740 & \\
\hline$B B$ & 0.640 & 0.568 & 0.212 & \\
\hline$E B$ & 0.816 & 0.962 & 0.924 & 0.358 \\
\hline \multicolumn{5}{|c|}{ Elevation coverage } \\
\hline$E E$ & 0.576 & 0.546 & 0.924 & \\
\hline$B B$ & 0.584 & 0.288 & 0.618 & \\
\hline$E B$ & 0.872 & 0.728 & 0.892 & 0.892 \\
\hline \multicolumn{5}{|l|}{ Boresight angle } \\
\hline$E E$ & 0.916 & 0.448 & 0.320 & \\
\hline$B B$ & 0.242 & 0.548 & 0.592 & \\
\hline$E B$ & 0.912 & 0.100 & 0.392 & 0.944 \\
\hline \multicolumn{5}{|l|}{ Temporal split } \\
\hline$E E$ & 0.378 & 0.208 & 0.796 & \\
\hline$B B$ & 0.788 & 0.020 & 0.852 & \\
\hline$E B$ & 0.370 & 0.580 & 0.476 & 0.232 \\
\hline \multicolumn{5}{|l|}{ Season split } \\
\hline$E E$ & 0.564 & 0.716 & 0.216 & \\
\hline$B B$ & 0.790 & 0.992 & 0.056 & \\
\hline$E B$ & 0.806 & 0.514 & 0.456 & 0.986 \\
\hline \multicolumn{5}{|l|}{ Focal plane $Q U$} \\
\hline$E E$ & 0.670 & 0.014 & 0.994 & \\
\hline$B B$ & 0.896 & 0.804 & 0.576 & \\
\hline$E B$ & 0.236 & 0.806 & 0.234 & 0.560 \\
\hline
\end{tabular}

value is, however, consistent with expectations from uniformly distributed PTEs over the 60 polarization-only jackknives. Figure 8 shows that the PTEs are consistent with a uniform distribution between zero and one.

The polarization jackknife tests are the most powerful probe of the accuracy of the noise model. In addition to the $\chi^{2}$ tests, we have also expressed the jackknife spectra as band power deviations with respect to the mean of signal-only simulations. The sum of the band power deviations in each jackknife spectrum provides an additional and more precise gauge of the correct estimation of instrument noise. We have verified that the band power deviation sums in the data are consistent with signalplus-noise simulations and are not systematically biased high or low, thus confirming that the noise levels are correctly estimated. Furthermore, we have probed the sensitivity to systematic variations in the modeled noise amplitude over a range that is comparable to the $\mathrm{S} / \mathrm{N}$ of the sum and difference timestreams, and find no significant changes in the jackknife spectra. The relative insensitivity of the jackknifes to the amplitude of the noise model validates the procedure described in Section 6.1.

In contrast to the polarization data, the temperature data display significant jackknife failures. There is an excess of small PTE values in the $T E$ and $T B$ jackknives, and most of the $T T$ jackknife PTEs are smaller than 0.002, which is the resolution from 500 simulations. The $T T, T E$, and $T B$ jackknife PTEs are therefore not listed in Table 1. We attribute these failures to the fact that BICEP's temperature maps have high $\mathrm{S} / \mathrm{N}$ (see TT plot in Figure 4), and the jackknives are therefore extremely sensitive to small gain calibration errors or imperfections in modeling and subtracting unpolarized atmospheric emission. (As described in Section 6.1, the $\leqslant 10 \% \mathrm{~S} / \mathrm{N}$ in the pair-sum timestreams is a known imperfection of the BICEP noise model for temperature data.) Although the $T T, T E$, and $T B$ jackknife failures are statistically significant, Figure 7 illustrates that the amplitudes of the jackknife spectra are small compared to both the amplitude and errors of the signal spectra. The magnitudes of the $T T$ and $T E / T B$ jackknife band powers are typically $1-10 \mu \mathrm{K}^{2}$ and $0.1-1 \mu \mathrm{K}^{2}$, respectively. In all cases, the error bars of the non-jackknife spectra are at least a factor of 10 larger, except in the highest $\ell$ bin.

We have performed the same jackknives with both analysis pipelines, and the results are in excellent agreement. The alternate pipeline confirms that the polarization data pass the $\chi^{2}$ tests, and although the $T T, T E$, and $T B$ spectra do not pass, the amplitudes of the jackknife spectra are small.

\section{SYSTEMATIC UNCERTAINTIES}

Systematic errors that arise from uncertainties in instrument characterization can be separated into two categories: (1) errors that mix temperature, $E$-mode, and $B$-mode polarization; and (2) calibration uncertainties that affect only the scaling or amplitude of the spectra. In this section, we describe the dominant sources of systematic error in BICEP and the expected impact on the power spectra; these systematics are summarized in Figure 9. A complete description of all potential BICEP systematics and the methodology for propagating the errors to the power spectra is given in the accompanying instrument characterization paper (Takahashi et al. 2010).

\subsection{Temperature and Polarization Mixing}

We are mostly concerned with systematic errors that mix the bright temperature signal into polarization and thus induce a false $B$-mode signal. We define a benchmark for each systematic error such that the false $B$-mode amplitude is no greater than the peak of the inflationary $B B$ spectrum. For BICEP's target of $r=0.1$, this requirement corresponds to $\ell(\ell+1) C_{\ell}^{B B} /(2 \pi)=$ $7 \times 10^{-3} \mu \mathrm{K}^{2}$ at $\ell \sim 100$.

The primary source of potential temperature leakage into polarization is differential gains within PSB pairs. Gain mismatch effects can also arise from other systematics, such as errors in the bolometer transfer functions, which act as frequency-dependent gains. We have characterized the common-mode rejection of PSBs by examining the cross-correlation of pair-sum and pairdifference maps. There is no statistically significant evidence for gain mismatch in the data, and we set an upper limit of $\Delta\left(g_{1} / g_{2}\right) /\left(g_{1} / g_{2}\right)<1.1 \%$ on differential gains.

A second source of potential temperature and polarization mixing is beam mismatch within PSB pairs. We describe beam mismatch with three quantities: differential beam size, pointing, and ellipticity. Of these three, the dominant effect in BICEP is differential pointing, which is stable over time and has been measured with a median amplitude of $\left(\mathbf{r}_{1}-\mathbf{r}_{2}\right) / \bar{\sigma}=1.3 \pm 0.4 \%$, where $\bar{\sigma}$ is the average Gaussian beam size within a pair of PSBs. Measured upper limits on differential size and ellipticity are negligible.

Most systematic errors interact with the scan strategy in complex ways, and the exact effects on the power spectra can be computed only through signal simulations. We follow the formalism presented in E. F. Hivon \& N. Ponthieu (2010, in preparation) and Shimon et al. (2008) to calculate the expected level of false $B B$ in BICEP. Starting from synfast maps with $r=0$, Equation (13) is used to generate simulated signal timestreams that include the effects of gain mismatch and differential pointing. The timestreams are filtered and co-added into maps, and the amplitude of the $B B$ power spectrum at $\ell \sim 100$ is compared with the $7 \times 10^{-3} \mu \mathrm{K}^{2}$ benchmark. 


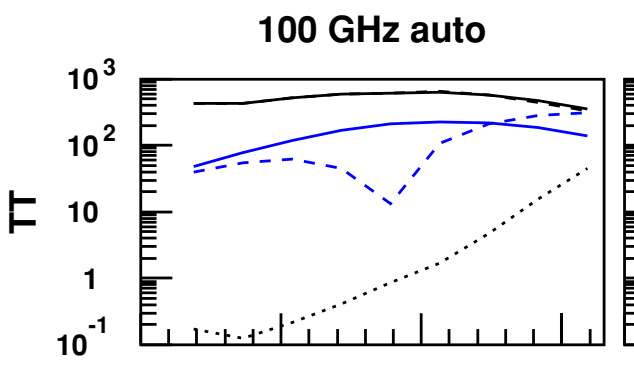

\section{$150 \mathrm{GHz}$ auto}
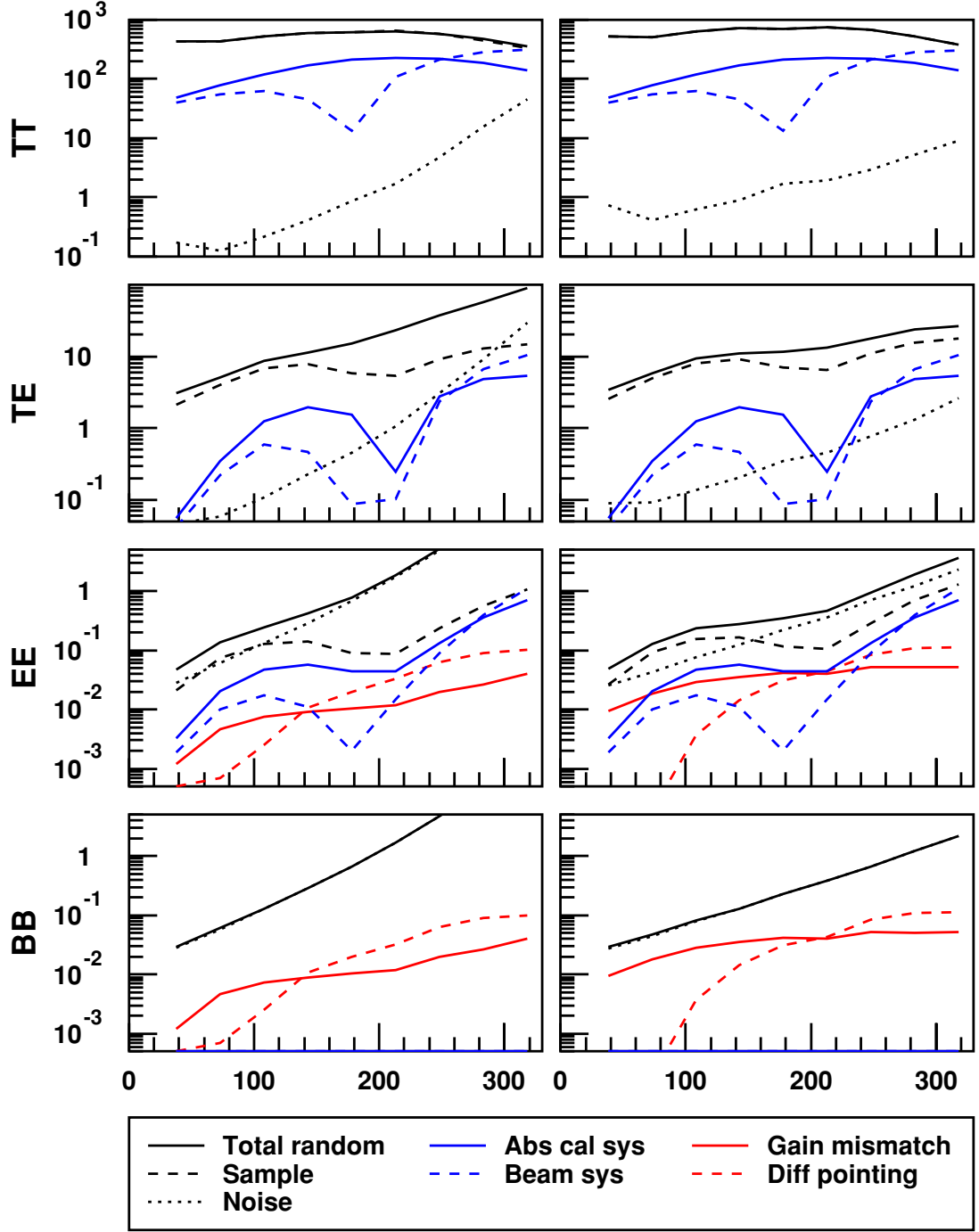

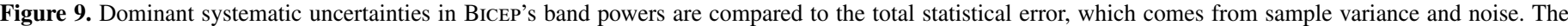

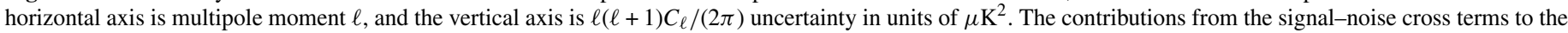

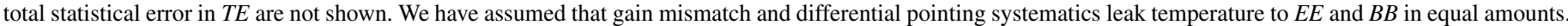

In the differential gain simulations, we randomly assign $1.1 \%$ rms gain mismatch to PSB pairs, and we find that the expected false $B B$ amplitude is $7.4 \times 10^{-3} \mu \mathrm{K}^{2}$ and $2.9 \times 10^{-2} \mu \mathrm{K}^{2}$ at $\ell \sim 100$ for 100 and $150 \mathrm{GHz}$, respectively. Although this amplitude exceeds the $r=0.1$ benchmark, Figure 9 illustrates that it is small compared to the statistical error in this analysis of the two-year data. In a future analysis of the entire BICEP data set, we anticipate placing tighter constraints on PSB gain mismatch as the noise levels integrate down. With further work, we are confident that uncertainty in gain mismatch can be substantially reduced from the current $1.1 \%$.

Differential pointing has been precisely characterized for each of BICEP's PSB pairs, so we run signal simulations using the measured centroid offset vectors, rather than randomized distributions. The false $B B$ from differential pointing has an $\ell \sim 100$ amplitude of $2.7 \times 10^{-3} \mu \mathrm{K}^{2}$ and $4.2 \times 10^{-3} \mu \mathrm{K}^{2}$ at 100 and $150 \mathrm{GHz}$, respectively. These amplitudes are slightly smaller than the $r=0.1$ benchmark and are well below the noise level of the initial two-year data set. In a future analysis, it may be possible to use the measured centroid offsets to correct for systematic effects.
We emphasize that, in addition to the differential gain and pointing discussed here, most uncertainties in instrument characterization create false positive $B B$ signal. The fact that BICEP's $B B$ spectra are consistent with zero and pass jackknives demonstrates that we have achieved sufficient control over systematic errors in this analysis. Furthermore, until a positive $B$-mode detection is made, the presence of systematic effects that produce spurious polarization could only make the reported $B B$ upper limits higher (more conservative) than they would be otherwise.

\subsection{Absolute Gain and Beam Uncertainty}

The scaling of the power spectra is determined by the absolute gain factors that convert detector units to temperature, and the $2 \%$ uncertainty in this gain (Section 3.2) translates into a $4 \%$ uncertainty in the power spectrum amplitude. The polarized spectra have additional amplitude uncertainty that arises from errors in the cross-polar leakage. A systematic error of $\Delta \epsilon=0.01$ corresponds to $3.9 \%$ amplitude uncertainty for polarization-only spectra $(E E, B B, E B)$ and $2.0 \%$ for temperature-polarization 
cross-spectra $(T E, T B)$. Therefore, the combined amplitude uncertainty $G^{X} \equiv \Delta C_{\ell}^{X} / C_{\ell}^{X}$ from gain and $\epsilon$ errors is $4 \%$ for $T T$, $4.5 \%$ for $T E / T B$, and $5.6 \%$ for $E E / B B / E B$. These uncertainties are shown in Figure 9, assuming $\Lambda$ CDM spectra with $r=0$.

For Gaussian beams, measurement errors in the beam widths introduce a fractional uncertainty,

$$
S_{\ell} \equiv \Delta C_{\ell}^{X} / C_{\ell}^{X}=e^{\sigma^{2} \ell(\ell+1)\left(\delta^{2}+2 \delta\right)}-1-\bar{S},
$$

in the power spectrum amplitude as a function of $\ell$. Here, $\sigma=\mathrm{FWHM} / \sqrt{8 \ln (2)}$, and $\delta$ is the fractional beam width error $\Delta \sigma / \sigma$. Because this band power uncertainty is degenerate with absolute gain error, we subtract the mean, $\bar{S}$, calculated over $56 \leqslant \ell \leqslant 265$, the angular scales over which we perform absolute calibration. For this analysis, we use average beam widths of 0.93 and 0.60 at 100 and $150 \mathrm{GHz}$, respectively. Although the distribution of beam widths in the focal plane varies by $\pm 3 \%$, the measurement precision is $\pm 0.5 \%$; we therefore expect the effective $\delta$ to lie somewhere in between. We calculate the maximum beam width error allowed by our calibration cross-spectra (Equation (2)), which are very close to flat, and we constrain $\delta<1.2 \%$ and $\delta<2.8 \%$ at 100 and $150 \mathrm{GHz}$, respectively. Figure 9 shows the expected power spectrum errors from these maximum allowed beam uncertainties, which are most likely conservative. The systematic error is smaller than the statistical error in all cases except the $T T$ spectra at high $\ell$, where the levels of statistical and beam systematic uncertainty are comparable.

\section{FOREGROUNDS}

The BicEP CMB region was chosen to have the lowest foreground dust emission for a field of that size, and we do not expect foreground contamination from dust or other sources to be significant at the current depth in the maps. To verify this, we estimate the levels of contamination in BICEP CMB data from three potential foreground sources: thermal dust emission, synchrotron radiation, and extragalactic radio point sources.

\subsection{Thermal Dust}

Polarized dust emission in the BICEP CMB field is estimated from "FDS Model 8" (Finkbeiner et al. 1999). We assume a 5\% polarized fraction, guided by a study of WMAP data that shows that high-latitude dust has a mean fractional polarization of $3.6 \%$ (Kogut et al. 2007). The dust temperature and polarization model is extrapolated to 100 and $150 \mathrm{GHz}$, and filtered according to BICEP's scan strategy. The resulting polarized dust emission is $\ell(\ell+1) C_{\ell} /(2 \pi)=9.6 \times 10^{-5} \mu \mathrm{K}^{2}$ and $6.1 \times 10^{-4} \mu \mathrm{K}^{2}$ at $\ell=100$ for 100 and $150 \mathrm{GHz}$, respectively. These values are 2 orders of magnitude below the $95 \%$ confidence level for upper limits on the $B B$ amplitude from BICEP (discussed in Section 13), as shown in Figure 10.

We have also tested for dust contamination in the BICEP maps by studying the cross power spectrum between FDS Model 8 maps and BICEP temperature and polarization maps. The cross power spectrum of the real data is consistent with the distribution from signal-plus-noise simulations, providing additional evidence that the BICEP maps contain no spatial correlation with the FDS dust maps.

\subsection{Synchrotron}

To estimate the polarized synchrotron emission in the BICEP field of view, we have used the WMAP Markov Chain Monte

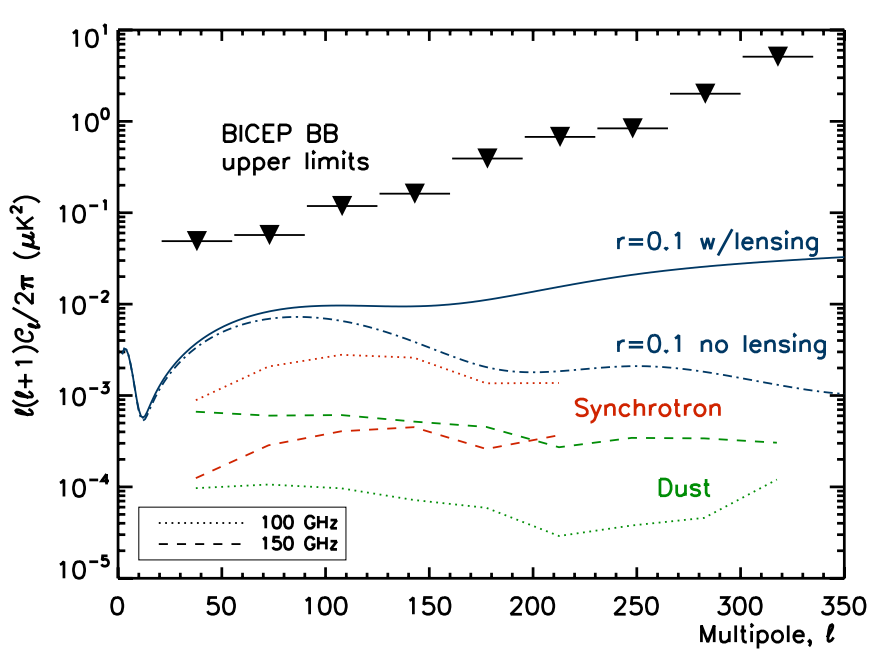

Figure 10. Expected levels of polarized dust and polarized synchrotron in the BICEP CMB field, assuming FDS Model 8 with 5\% polarization fraction and WMAP MCMC polarization maps extrapolated to 100 and $150 \mathrm{GHz}$. These estimated foreground levels are much lower than the $B B$ upper limits presented in Section 13. Theoretical $B B$ curves for $r=0.1$, with and without gravitational lensing, are also shown for comparison.

Carlo (MCMC) synchrotron maps (Gold et al. 2009), extrapolated to 100 and $150 \mathrm{GHz}$ using the mean spectral index of the BICEP field of view, calculated from the spectral index maps provided in the same analysis. As for the FDS dust maps, we filter the extrapolated 100 and $150 \mathrm{GHz}$ synchrotron maps with the BICEP scan strategy, and find the estimated level of polarized synchrotron emission at $\ell \sim 100$ to be $3 \times 10^{-3} \mu \mathrm{K}^{2}$ and $4 \times 10^{-4} \mu \mathrm{K}^{2}$ for 100 and $150 \mathrm{GHz}$, respectively, both below the level of BICEP sensitivity. Furthermore, the WMAP MCMC map has poor $\mathrm{S} / \mathrm{N}$ in regions far from the Galactic plane, and we have found that within the BICEP CMB field, the map is dominated by variance in the Monte Carlo fit (see Gold et al. 2009, for details). The estimated levels of polarized synchrotron emission in Figure 10 should therefore be viewed as conservative estimates or upper limits. We have also derived synchrotron estimates from WMAP K-band polarization maps and the temperature maps of Haslam et al. (1981), assuming a 30\% polarization fraction; both methods give synchrotron estimates that are lower than those from the WMAP MCMC maps.

Similarly to our analysis for thermal dust, we have studied the cross power spectrum of the synchrotron maps with the BICEP maps and found there to be no significant spatial correlation of the BICEP data with the synchrotron emission.

\subsection{Point Sources}

At degree-scale resolution, the BICEP maps do not show any obvious point source detections, so we rely on a combination of the 4.85 GHz Parkes-MIT-NRAO (PMN) survey (Wright et al. 1994), the WMAP point source catalog (Wright et al. 2009), and the ACBAR catalog (Reichardt et al. 2009) to search for point source contamination. We search for point source contamination by optimally filtering $\mathrm{CMB}$ and noise fluctuations out of the BICEP temperature map and determine the significance of the resulting pixel values by repeating the process with simulated maps of CMB with detector noise. Although the resulting maps have a few $2 \sigma$ detections at the suspected point source locations, there is no statistical evidence for point source contamination above the expected Gaussian distribution of noise. As a further test, we have simulated the effects of masking out the 27 ACBAR 
sources that lie within the BICEP field and have found it has no significant impact on the power spectra.

\subsection{Frequency Jackknife}

The CMB and foreground emission have different frequency dependence, so we can test for the presence of foreground contamination in BICEP data by performing a frequency jackknife. We difference the 100 and $150 \mathrm{GHz}$ maps, compute the power spectra, calculate $\chi^{2}$, and compare the results to signal-plusnoise simulations, as described in Section 8.1. The probabilities to exceed the $\chi^{2}$ values are $\{0.050,0.152,0.732\}$ for $E E, B B$, and $E B$, respectively. We find no evidence for foreground contamination in the frequency jackknives.

\section{COMBINED SPECTRA}

We combine the spectra from the different observing frequencies by taking a weighted average for each band power. To obtain the weights, we use signal-plus-noise simulations to calculate the covariance matrices from the various frequency combinations $(100,150,100 \times 150$, and $150 \times 100 \mathrm{GHz})$. There are three unique combinations for $T T, E E$, and $B B$, and four combinations for the other spectra. The weights are calculated from the row/column sums of the inverse of the covariance matrices. The error bars of the combined spectra are determined by applying the same combination weights to signal-plus-noise simulations. For fully noise-dominated spectra (such as $B B$ ), the error bars of the combined spectra improve by $10 \%-40 \%$ compared to the errors from $150 \mathrm{GHz}$ data alone.

As suggested by Bond et al. (2000), we apply a transformation

$$
Z_{b}=\ln \left(\mathscr{C}_{b}+x_{b}\right)
$$

to account for the fact that the probability of the true model value, given an observed band power, is offset-lognormally distributed. The offsets $x_{b}$ describe the noise power spectra on the sky (i.e., corrected for filter and beam bias) and are calculated from simulations. We calculate $x_{b}$ for the $T T, E E$, and $B B$ spectra, but we assume Gaussian distributions for the $T E, T B$, and $E B$ band powers since the values can be negative. The BICEP band powers, $x_{b}$ offsets, covariance matrices, and band power window functions are available online at http://bicep.caltech.edu.

Figure 11 shows a comparison of the frequency-combined spectra with a $\Lambda C D M$ model derived from WMAP five-year data. The power spectrum results are confirmed by the alternate analysis pipeline (open circles, Figure 11). BICEP contributes the first high $\mathrm{S} / \mathrm{N}$ polarization measurements around $\ell \sim 100$, as illustrated by Figure 12, which shows the $E E$ peak at $\ell \sim 140$ in greater detail; the $B B$ spectrum is overplotted for comparison. Figure 13 shows BICEP's $T E$ and $E E$ spectra, as well as the 95\% confidence upper limits on $B B$, in addition to other recent $C M B$ polarization data. To obtain the $B B$ upper limits, we apply offsetlognormal transformations to the band powers and integrate the positive portion of the band power probability distributions up to the $95 \%$ point. BICEP measures $E E$ in a multipole window that complements existing data from other experiments, and all nine band powers have $>2 \sigma$ significance. The constraints on $B B$ are the most powerful to date.

\section{CONSISTENCY WITH $\Lambda$ CDM}

The power spectra of the CMB are well described by a $\Lambda$ CDM model, which, in its simplest form, has six parameters that have been constrained by numerous experiments. We check the consistency of the BICEP band powers with this model by performing a $\chi^{2}$ test. We start by using CAMB to calculate theoretical power spectra, using $\Lambda \mathrm{CDM}$ parameters derived from WMAP five-year data (and $r=0$ ), and we then compute expected band power values, $\mathscr{C}_{b}^{X}$, using the band power window functions described in Section 6.5. Absolute gain and beam systematic errors $\left(G_{X}\right.$ and $S_{b}$, as described in Section 9.2) are included by adding their contributions to the band power covariance matrix, $M_{a b}^{X}$ :

$$
\mathcal{M}_{a b}^{X}=M_{a b}^{X}+\left(G^{X}\right)^{2} \mathscr{C}_{a}^{X} \mathscr{C}_{b}^{X}+S_{a} S_{b} \mathscr{C}_{a}^{X} \mathscr{C}_{b}^{X}
$$

The $S_{b}$ factors are formed from linear combinations of the four frequencies $(100 \mathrm{GHz}$ auto, $150 \mathrm{GHz}$ auto, $100 \times 150,150 \times$ 100 ), using the weights described in Section 11. Because $M_{a b}^{X}$ is obtained from a limited number of simulations, the far offdiagonal terms are dominated by noise; we therefore use only the main and first two off-diagonal terms of $M_{a b}^{X}$ in this calculation. (We have tested that results are essentially unchanged including one, two, or all off-diagonal terms.) For each power spectrum, the observed and theoretical band powers are compared by evaluating

$$
\chi^{2}=\left[\hat{\mathscr{C}}^{X}-\mathscr{C}^{X}\right]^{\top}\left(\mathcal{M}^{X}\right)^{-1}\left[\hat{\mathscr{C}}^{X}-\mathscr{C}^{X}\right]
$$

over the nine bins that span $21 \leqslant \ell \leqslant 335$. In the case of the $T T, E E$, and $B B$ spectra, offset-lognormal transformations,

$$
\begin{gathered}
\hat{Z}_{b}^{X}=\ln \left(\hat{\mathscr{C}}_{b}^{X}+x_{b}^{X}\right), \\
Z_{b}^{X}=\ln \left(\mathscr{C}_{b}^{X}+x_{b}^{X}\right), \\
\left(D_{a b}^{X}\right)^{-1}=\left(\mathcal{M}_{a b}^{X}\right)^{-1}\left(\hat{\mathscr{C}}_{a}^{X}+x_{a}^{X}\right)\left(\hat{\mathscr{C}}_{b}^{X}+x_{b}^{X}\right),
\end{gathered}
$$

are applied to the data, expected band powers, and inverse covariance matrix, and $\chi^{2}$ is calculated using the transformed quantities.

We perform the same calculations for a set of 500 signal-plusnoise simulations, and the simulated $\chi^{2}$ distributions are used to determine the probabilities to exceed the $\chi^{2}$ values of the data. The $\chi^{2}$ and PTE values are listed in Figure 11, which shows a comparison of our data with the $\Lambda$ CDM model. The BICEP data are consistent with $\Lambda \mathrm{CDM}$, and this result is confirmed by the alternate analysis pipeline.

\section{CONSTRAINT ON TENSOR-TO-SCALAR RATIO FROM $B B$}

BICEP was designed with the goal of measuring the $B B$ spectrum at degree angular scales in order to constrain the tensor-to-scalar ratio $r$. We define $r=\Delta_{h}^{2}\left(k_{0}\right) / \Delta_{R}^{2}\left(k_{0}\right)$, where $\Delta_{h}^{2}$ is the amplitude of primordial gravitational waves, $\Delta_{R}^{2}$ is the amplitude of curvature perturbations, and we choose a pivot point $k_{0}=0.002 \mathrm{Mpc}^{-1}$. The tightest published upper limit is $r<0.22$ at $95 \%$ confidence and is derived from a combination of the WMAP five-year measurements of the $T T$ power spectrum at low $\ell$ with measurements of Type Ia supernovae and baryon acoustic oscillations (Komatsu et al. 2009).

As a method for constraining $r$, a direct measurement of the $B B$ spectrum has two advantages. First, measurements of $T T$ are ultimately limited by cosmic variance at large angular scales, and the temperature data from WMAP have already reached that 

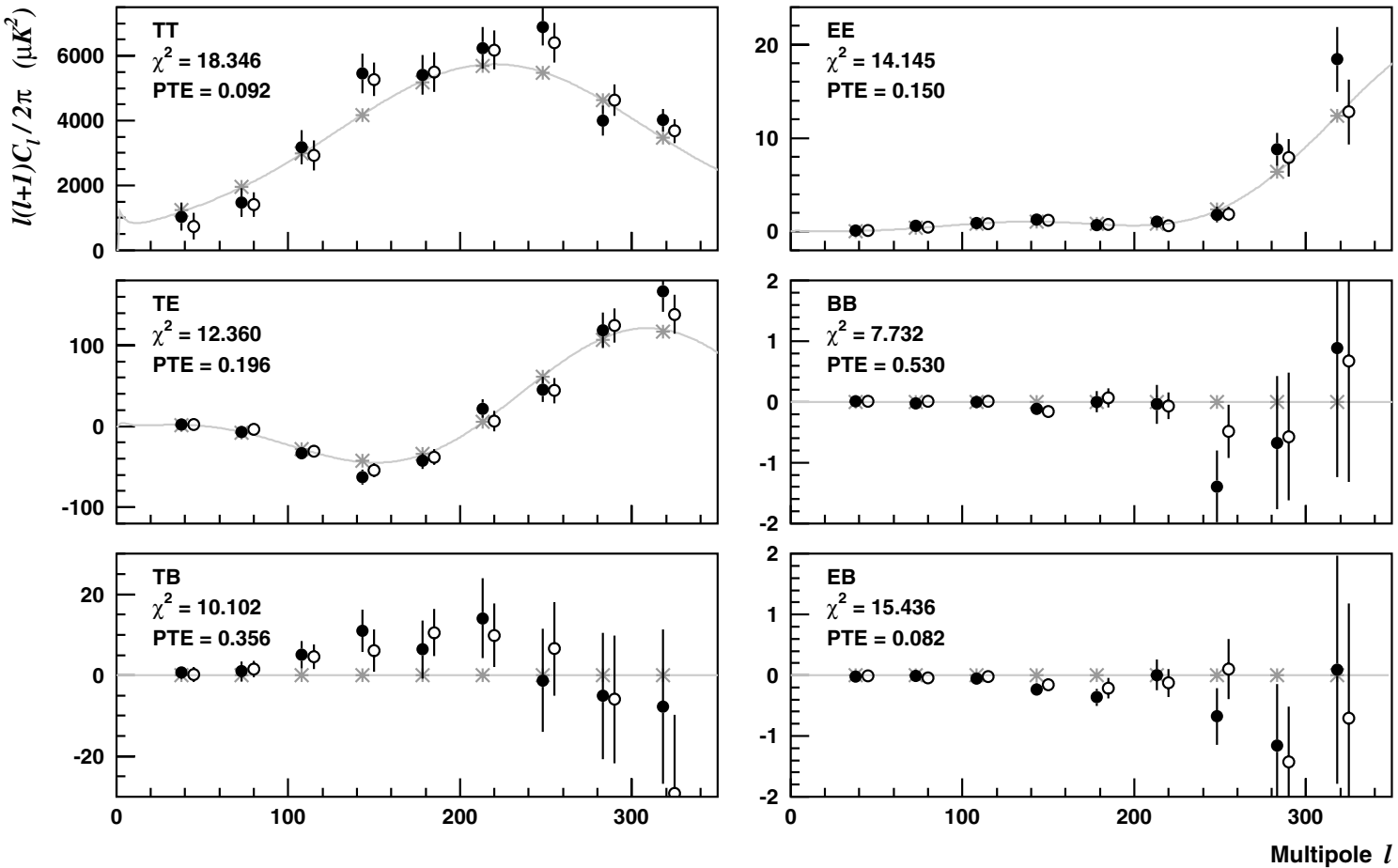

Figure 11. BICEP's combined power spectra (black points) are in excellent agreement with a $\Lambda$ CDM model (gray lines) derived from $W M A P$ five-year data. The $\chi^{2}$ (for nine degrees of freedom) and PTE values from a comparison of the data with the model are listed in the plots. The asterisks denote theoretical band power expectation values. Power spectrum results from the alternate analysis pipeline are shown by the open circles and are offset in $\ell$ for clarity.

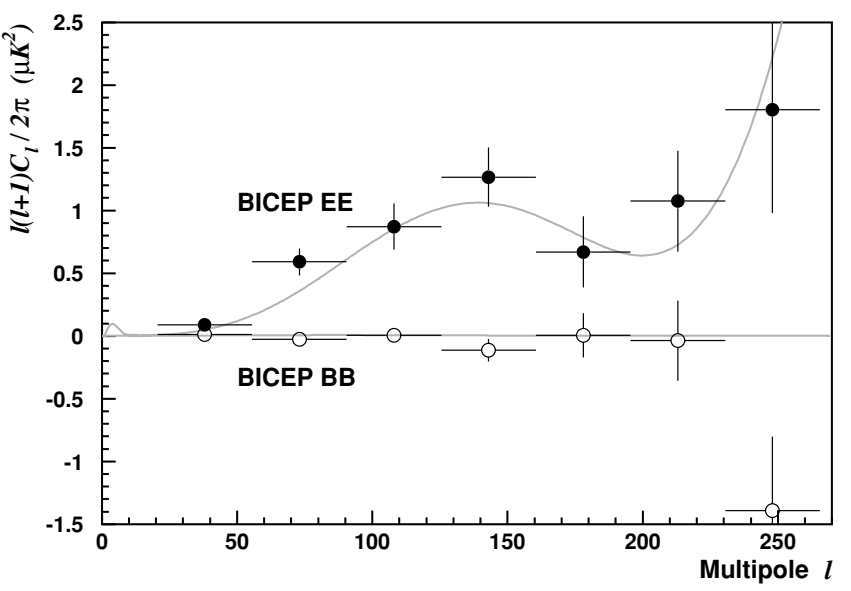

Figure 12. Bicep measures $E E$ polarization (black points) with high $\mathrm{S} / \mathrm{N}$ at degree angular scales. The $B B$ spectrum (open circles) is overplotted and is consistent with zero. Theoretical $\Lambda \mathrm{CDM}$ spectra (with $r=0.1$ ) are shown for comparison.

limit. Second, $r$ constraints from $T T$ are limited by parameter degeneracies; in particular, there is a strong degeneracy with the scalar spectral index $n_{s}$. The inflationary $B B$ spectrum, in contrast, suffers little from parameter degeneracies at the angular scales that BICEP probes, and the $B B$ amplitude depends primarily on $r$.

To determine a constraint on $r$ from BICEP's $B B$ spectrum, we compare the measured band powers, $\hat{\mathscr{C}}_{b}^{B B}$, to a template $B B$ curve and vary the amplitude of the inflationary component, assuming that it simply scales with $r$. The template $B B$ curves are calculated using fixed $\Lambda \mathrm{CDM}$ parameters derived from WMAP five-year data and a wide range of trial $r$ values. We include a constant $B B$ component from gravitational lensing, although the contribution is negligible at low multipoles. From the template $B B$ curves, we compute the expected band powers, $\mathscr{C}_{b}^{B B}(r)$. We apply offset-lognormal transformations to the data, expected band powers, and covariance matrix (Equations (25)-(27)), and we calculate

$$
\chi^{2}(r)=\left[\hat{\mathbf{Z}}^{B B}-\mathbf{Z}^{B B}(r)\right]^{\top}\left[\mathbf{D}^{B B}(r)\right]^{-1}\left[\hat{\mathbf{Z}}^{B B}-\mathbf{Z}^{B B}(r)\right]
$$

at each trial $r$ value. The likelihood is then

$$
L \propto \frac{1}{\sqrt{\operatorname{det}\left[\mathbf{D}^{B B}(r)\right]}} e^{-\chi^{2}(r) / 2} .
$$

Figure 14 (left panel) shows that the maximum likelihood value obtained from BICEP data is $r=0.02_{-0.26}^{+0.31}$. For comparison, the central panel shows the maximum likelihood $r$ values from 500 signal-plus-noise simulations with $r=0$ input spectra. We calculate the upper limit on $r$ by integrating the positive portion of the likelihood up to the $95 \%$ point, and we find that the BICEP $B B$ spectrum constrains $r<0.72$ at $95 \%$ confidence. This constraint is consistent with those obtained from simulations (Figure 14, right panel).

We have cross-checked the BICEP $r$ constraint with two methods. First, the alternate analysis pipeline yields very similar estimates of $r$, adding confidence to our result. Second, we have generated 100 signal-plus-noise simulations using input spectra corresponding to $r=0.72$, and we have calculated $r$ likelihood curves for each of these realizations. Figure 15 shows the histogram of maximum likelihood $r$ values, which peaks as expected around the input value of 0.72 , confirming our pipeline recovers an unbiased estimate of $r$. Only two of 100 simulations have maximum likelihood $r$ values that fall below the data; this simple alternative statistic to the Bayesian 95\% upper limit suggests at a similar level of confidence that the BICEP data excludes the $r=0.72$ hypothesis. 


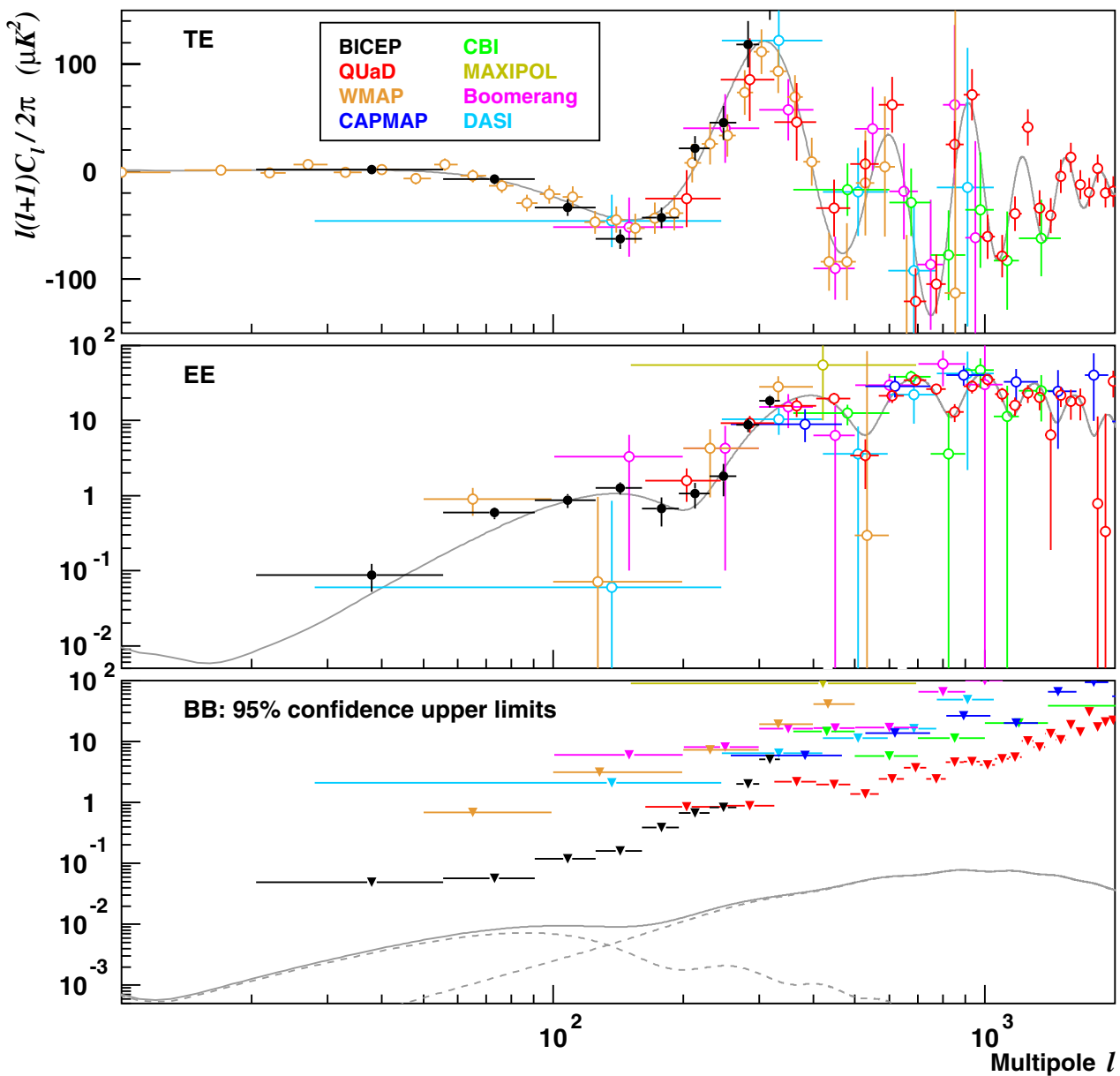

Figure 13. BICEP's $T E, E E$, and $B B$ power spectra complement existing data from other CMB polarization experiments (Leitch et al. 2005; Montroy et al. 2006; Piacentini et al. 2006; Sievers et al. 2007; Wu et al. 2007; Bischoff et al. 2008; Nolta et al. 2009; Brown et al. 2009). Theoretical spectra from a $\Lambda$ CDM model with $r=0.1$ are shown for comparison; the $B B$ curve is the sum of the inflationary and gravitational lensing components. At degree angular scales, BICEP's constraints on $B B$ are the most powerful to date.
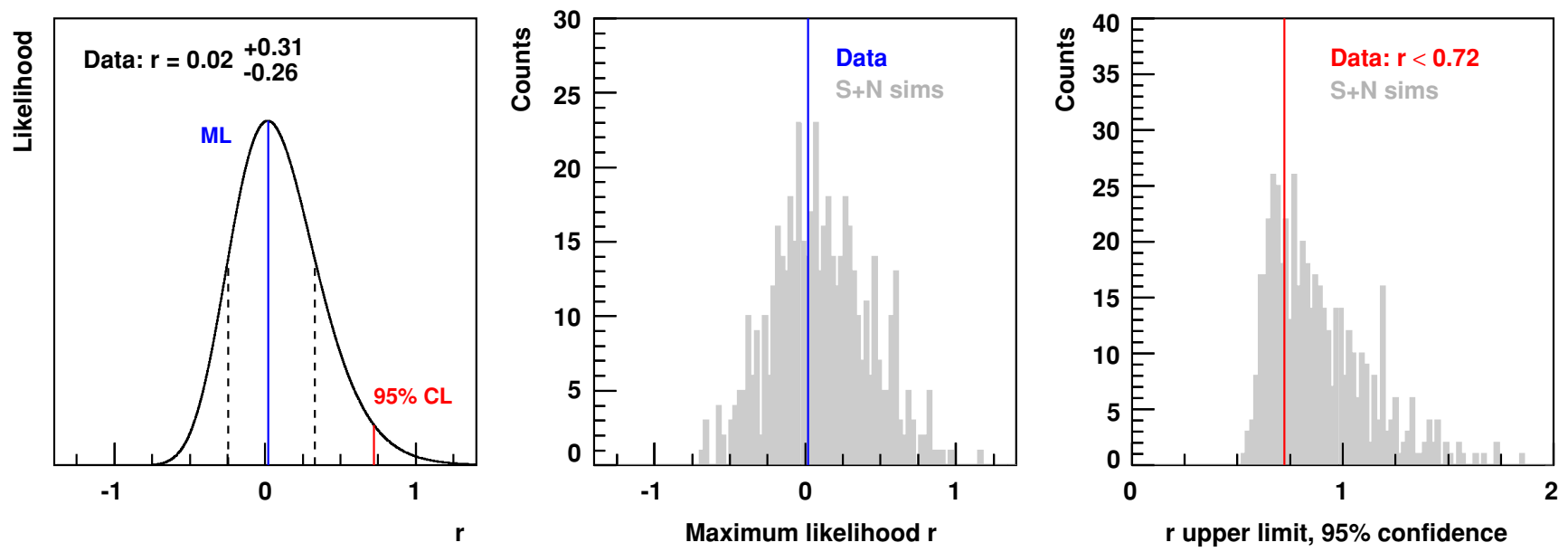

Figure 14. $r$ likelihood function calculated from the BICEP $B B$ spectrum is shown in the left panel and peaks at a value of $r=0.02$. For comparison, a histogram of maximum likelihood $r$ values derived from 500 signal-plus-noise simulations (with $r=0$ input) is shown in the central panel. In the right panel, we derive $95 \%$ confidence upper limits on $r$ from the both the simulated and real likelihoods. The data yield an upper limit of $r<0.72$, which lies within the simulated distribution.

While limits on $r$ derived from CMB data are still driven by the WMAP measurement of the TT power spectrum, BICEP's limits on the amplitude of the $B B$ spectrum are an order of magnitude more powerful than any previous measurement. The improvement in the power of $B B$ to constrain $r$ is illustrated by repeating the above analysis using WMAP $B B$ data, where we obtain a limit of $r<6$, versus the BICEP constraint of $r<0.72$.

\section{CONCLUSIONS}

Motivated by the exciting possibility of detecting, albeit indirectly, the gravitational wave background due to inflation, many efforts are underway to develop the instrumentation and methods necessary to search for the $B$-mode component of CMB polarization at degree angular scales. In this paper, we 


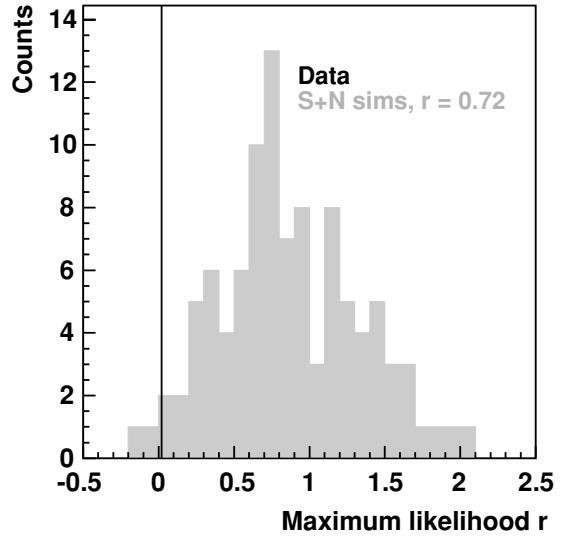

Figure 15. Maximum likelihood $r$ values are shown for 100 signal-plus-noise simulations with input spectra for $r=0.72$, a value chosen to match BICEP's $95 \%$ upper limit. As expected, the distribution peaks around 0.72 , showing the BICEP pipeline recovers an unbiased estimate of $r$. Only two of the 100 simulations fall below $r=0.02$, the maximum likelihood value derived from BICEP data, offering an alternative demonstration that $r=0.72$ is strongly disfavored by the BICEP data.

have presented initial results from BICEP, the first experiment optimized specifically to search for the inflationary $B$-mode signal. Analysis of a subset of the first two years of observations provides the most sensitive measurement to date of $\mathrm{CMB}$ polarization at degree angular scales. The $T T, T E$, and $E E$ angular power spectra are measured with high $\mathrm{S} / \mathrm{N}$ the $T B$, $B B$, and $E B$ spectra are consistent with zero. The spectra are consistent with a $\Lambda C D M$ model using parameters derived from $W M A P$ five-year data. The polarization data pass all of the statistical tests that we have been able to devise. Furthermore, the results have been cross-checked by two independent analysis pipelines, whose agreement is excellent at all stages of the data processing, including maps, power spectra, jackknives, and comparisons with theoretical models. A detailed study of BICEP's potential systematic errors indicate that all systematic effects are below the level of the statistical errors and are not limiting factors in the analysis.

BICEP has detected, for the first time, the first peak in the angular power spectrum of the $E$-mode polarization. The combination of these results from BICEP and of results from its sister experiment, QUAD, confirm with significant precision the theoretical prediction for the shape and amplitude of the $E$-mode spectrum on angular scales that span its first six peaks. In addition, both experiments have shown that there is no $B$-mode component comparable in amplitude to the $E$-mode component.

Because BICEP is designed specifically to probe the degree angular scales at which the inflationary $B$-mode signature peaks, BICEP's upper limits on the $B$-mode angular power spectrum provide the first meaningful constraint on the inflationary gravitational wave background to come directly from CMB $B$-mode polarization, $r=0.02_{-0.26}^{+0.31}$ or $r<0.72$ at $95 \%$ confidence. Because this limit is dominated by statistical noise, constraints on $r$ may be expected to improve in proportion to observing time (not its square root), and it is likely that analysis of the remaining data from the three seasons of observing will provide improved limits on $r$. Future BICEP analyses will include the full data set, explore relaxing the conservative cuts employed for the initial analysis, and optimize the power spectrum recovery, all of which will strengthen BICEP constraints on $r$ significantly.

We dedicate this work to the memory of Andrew Lange, whose tragic and untimely death has deeply pained us all. He was and always will be an inspiration to us, and he is immeasurably missed.

BICEP is supported by NSF Grant No. OPP-0230438, Caltech President's Discovery Fund, Caltech President's Fund PF-471, JPL Research and Technology Development Fund, and the late J. Robinson. We thank the South Pole Station staff for helping make our observing seasons a success. We also thank Joanna Dunkley, Nathan Miller, and our colleagues in ACBAR, BOOMERANG, QUAD, BoLOCAM, SPT, and WMAP for advice and helpful discussions, and Kathy Deniston for logistical and administrative support. We gratefully acknowledge support of individual team members by the NASA Graduate Fellowship program (H.C.C.), NSF PECASE Award No. AST-0548262 (B.G.K.), the John B. and Nelly Kilroy Foundation (J.M.K.), the U.S. DOE contract to SLAC No. DE-AC02-76SF00515 (C.L.K. and J.E.T.), KICP (C.P. and C.S.), and the NASA Science Mission Directorate via the US Planck Project (G.R.).

\section{REFERENCES}

Bischoff, C., et al. 2008, ApJ, 684, 771

Bond, J. R., Jaffe, A. H., \& Knox, L. 2000, ApJ, 533, 19

Brown, M. L., et al. 2009, ApJ, 705, 978

Chon, G., Challinor, A., Prunet, S., Hivon, E., \& Szapudi, I. M. 2004, MNRAS, 350,914

Dodelson, S., et al. 2009, arXiv:0902.3796

Finkbeiner, D. P., Davis, M., \& Schlegel, D. J. 1999, ApJ, 524, 867

Friedman, R. B., et al. 2009, ApJ, 700, L187

Gold, B., et al. 2009, ApJS, 180, 265

Górski, K. M., Hivon, E., Banday, A. J., Wandelt, B. D., Hansen, F. K., Reinecke, M., \& Bartelmann, M. 2005, ApJ, 622, 759

Haslam, et al. 1981, A\&A, 100, 209

Hinshaw, G., et al. 2009, ApJS, 180, 225

Jones, W. C., Bhatia, R., Bock, J. J., \& Lange, A. E. 2003, Proc. SPIE, 4855, 227

Jones, W. C., et al. 2006, ApJ, 647, 823

Jones, W. C., et al. 2007, A\&A, 470, 771

Kamionkowski, M., Kosowsky, S., \& Stebbins, A. 1997, Phys. Rev. Lett., 78, 2058

Kogut, A., et al. 2007, ApJ, 665, 335

Komatsu, E., et al. 2009, ApJS, 180, 330

Kovac, J. M., Leitch, E. M., Pryke, C., Carlstrom, J. E., Halverson, N. W., \& Holzapfel, W. L. 2002, Nature, 420, 772

Leitch, E. M., Kovac, J. M., Halverson, N. W., Carlstrom, J. E., Pryke, C., \& Smith, M. W. E. 2005, ApJ, 624, 10

Lewis, A., Challinor, A., \& Lasenby, A. 2000, ApJ, 538, 473

Montroy, T. E., et al. 2006, ApJ, 647, 813

Nolta, M. R., et al. 2009, ApJS, 180, 296

Piacentini, F., et al. 2006, ApJ, 647, 833

Polnarev, A. G. 1985, Sov. Astron., 29, 607

Pryke, C., et al. 2009, ApJ, 692, 1247

Reichardt, C. L., et al. 2009, ApJ, 694, 1200

Seljak, U., \& Zaldarriaga, M. 1997, Phys. Rev. Lett., 78, 2054

Shimon, M., et al. 2008, Phys. Rev. D, 77, 083003

Sievers, J. L., et al. 2007, ApJ, 660, 976

Sievers, J. L., et al. 2009, arXiv:0901.4540

Takahashi, Y. D., et al. 2010, ApJ, 711, 1141

Wright, A. E., et al. 1994, ApJS, 91, 111

Wright, E. L., et al. 2009, ApJS, 180, 283

Wu, J. H. P., et al. 2007, ApJ, 665, 55

Yoon, K. W., et al. 2006, Proc. SPIE, 6275, 62751K 